\title{
A COLLOCATION METHOD FOR SOLVING NONLINEAR VOLTERRA INTEGRO-DIFFERENTIAL EQUATIONS OF NEUTRAL TYPE BY SIGMOIDAL FUNCTIONS
}

\author{
DANILO COSTARELLI AND RENATO SPIGLER
}

Communicated by Hermann Brunner

\begin{abstract}
A numerical collocation method is developed for solving nonlinear Volterra integro-differential equations (VIDEs) of the neutral type, as well as other non-standard and classical VIDEs. A sigmoidal functions approximation is used to suitably represent the solutions. Special computational advantages are obtained using unit step functions, and important applications can also be obtained by using other sigmoidal functions, such as logistic and Gompertz functions. The method allows one to obtain a simultaneous approximation of the solution to a given VIDE and its first derivative, by means of an explicit formula. A priori as well as a posteriori estimates are derived for the numerical errors, and numerical examples are given for the purpose of illustration. A comparison is made with the classical piecewise polynomial collocation method as for accuracy and CPU time.
\end{abstract}

1. Introduction. In $[\mathbf{1 9}]$, a collocation method was introduced for solving nonlinear Volterra integral equations of the second kind, using the results established in [18], suited to approximate functions in certain classes by sigmoidal functions. Sigmoidal functions were then used in [21] to numerically solve balance laws.

In this paper, we use the sigmoidal functions approximation to develop a new collocation method for solving nonlinear Volterra integro-

2010 AMS Mathematics subject classification. Primary 65R20, 41A30, 45D05, $45 \mathrm{G} 10$.

Keywords and phrases. Collocation methods, sigmoidal function approximation, unit step functions, logistic functions, Gompertz functions, nonlinear Volterra integro-differential equations, neutral type integro-differential equations, non standard integro-differential equations.

This work was supported, in part, by the GNAMPA and the GNFM of the Italian INdAM.

Received by the editors on November 19, 2012, and in revised form on March 5, 2013. 
differential equations (VIDEs) of neutral type, of the form

$$
y^{\prime}(t)=f(t, y(t))+\int_{a}^{t} K\left(t, s, y(s), y^{\prime}(s)\right) d s, \quad y(a)=y_{0},
$$

for $t \in I:=[a, b]$, where $f$ and $K$ are sufficiently smooth given functions, see $[\mathbf{1 0}, \mathbf{1 4}, \mathbf{3 4}, \mathbf{3 5}]$. The method is also suited for solving classical VIDEs, i.e., equations where the integral term in (I) does not depend on $y^{\prime}(s)$, as well as non-standard VIDEs, i.e., classical equations where the integral term depends in addition on $y(t)$, see $[\mathbf{9}, \mathbf{3 3}]$. The most known example of a non-standard VIDE is perhaps given by the logistic equation with a memory term, see $[\mathbf{5}, \mathbf{6 ,} \mathbf{2 2}]$. Collocation methods are widely used to solve integral equations, see, e.g., $[\mathbf{2}, \mathbf{4}$, $9,12,13]$.

Sigmoidal functions arise in the theory of neural networks, where they play the role of activation functions of artificial neurons forming the network, see, e.g., $[\mathbf{1}, \mathbf{2 0}, \mathbf{2 7}, \mathbf{3 0}, \mathbf{3 2}, \mathbf{3 6}]$. Recall that a function $\sigma: \mathbf{R} \rightarrow \mathbf{R}$ is termed a sigmoidal function whenever $\lim _{x \rightarrow-\infty} \sigma(x)=0$ and $\lim _{x \rightarrow+\infty} \sigma(x)=1$. Neural networks are widely used in approximation theory, and many important papers have been written in recent years concerning this subject, see, e.g., $[\mathbf{7}, \mathbf{2 3}, \mathbf{2 4}]$. Results concerning the rate of this kind of approximation were given in $[\mathbf{7}, \mathbf{1 5}, \mathbf{2 4}, \mathbf{2 5}$, 28, 29].

Our collocation method consists of first approximating $y^{\prime}$ by neural networks by unit step (Heaviside) sigmoidal functions, $H$, of the form

$$
\sum_{k=0}^{N} \alpha_{k} H\left(t-t_{k}\right),
$$

where the coefficients $\alpha_{k}$ have to be determined, and $t_{k}$ 's are suitable nodes. Upon integrating (II), we then obtain an approximation for $y$ as well. At this point, replacing $y$ and $y^{\prime}$ in (I) with their approximations, and evaluating the equations at suitable collocation points on the interval $I$, we can determine the unknowns $\alpha_{k}$.

The choice of using unit step functions allows one to solve a large class of integral equations with some computational advantages. In particular, we can determine an explicit formula for calculating the coefficients $\alpha_{k}$. In this way, an analytical representation for the 
collocation solutions can be obtained, along with the analytical form of (II). Moreover, we can show that approximate solutions can also be given in terms of other sigmoidal functions, such as for instance logistic and Gompertz functions.

The numerical errors made approximating $y$ and $y^{\prime}$ are analyzed, and some a priori as well as a posteriori estimates are derived for such errors. A number of numerical examples are presented, and the results are compared with those obtained by the classical piecewise polynomial collocation method. The collocation method based on sigmoidal functions developed here seems to be competitive regarding the CPU time it requires. As for its accuracy, it performs better than piecewise polynomial collocation when integro-differential equations with weakly singular kernels are involved.

Here is the outline of the paper. In Section 2, we review some existing approximation results based on superposition of sigmoidal functions. In Section 3, our collocation method is described in detail, and it is shown that, for every given VIDE, our method yields a unique collocation solution. In Section 4, the numerical errors affecting the method are analyzed, and in subsection 4.1 some a priori error estimates are given. In subsection 4.2, some a posteriori error estimates are also provided. Finally, in Section 5, numerical examples are given to illustrate the performance of the method.

2. Approximation by bounded sigmoidal functions. Neural networks are widely used in approximation theory, where they play the role of universal approximators $[16,18,23,30,32,36]$. Usually, the activation function of the network is a sigmoidal function. Recall that $\sigma: \mathbf{R} \rightarrow \mathbf{R}$ is termed a sigmoidal function, provided that $\lim _{x \rightarrow-\infty} \sigma(x)=0$ and $\lim _{x \rightarrow+\infty} \sigma(x)=1$. Sometimes, boundedness, continuity and/or monotonicity are prescribed in addition.

In what follows, $C^{0}[a, b]$ will denote the set of all continuous functions $f:[a, b] \rightarrow \mathbf{R}$. In $[\mathbf{1 8}]$, the following result, concerning uniform approximation by sigmoidal functions, was proven using constructive arguments.

Theorem 2.1. Let $\sigma$ be a bounded sigmoidal function, and let $f \in C^{0}[a, b]$ be fixed. For every $\varepsilon>0$, there exist $N \in \mathbf{N}^{+}$and $w>0$ 
(depending on $N$ and $\sigma$ ), such that, if

$$
\begin{aligned}
\left(G_{N} f\right)(t):= & \sum_{k=1}^{N}\left[f\left(t_{k}\right)-f\left(t_{k-1}\right)\right] \sigma\left(w\left(t-t_{k}\right)\right) \\
& +f\left(t_{0}\right) \sigma\left(w\left(t-t_{-1}\right)\right)
\end{aligned}
$$

for $t \in[a, b], h:=(b-a) / N$, and $t_{k}:=a+k h, k=-1,0,1, \ldots, N$, then

$$
\left\|G_{N} f-f\right\|_{\infty}:=\sup _{t \in[a, b]}\left|\left(G_{N} f\right)(t)-f(t)\right|<\varepsilon
$$

We emphasize that the continuity of $\sigma$ is not required. In particular, assuming $\sigma$ continuous, Theorem 2.1 can be viewed as a density result for the set of all functions of the form (1), with respect to the uniform topology of $C^{0}[a, b]$.

Remark 2.2. The form of the coefficients in (1) is independent of the specific choice of $\sigma$. Therefore, various approximations of $f$ could be provided using different sigmoidal functions, keeping the same coefficients.

An important example of bounded sigmoidal functions is given by the well-known logistic function, defined as $\sigma_{\ell}(t):=\left(1+e^{-t}\right)^{-1}, t \in \mathbf{R}$. Logistic functions are largely used in biology, physics, and demography (see, e.g., $[\mathbf{8}, \mathbf{2 6}]$ ). Now, using Theorem 2.1 and choosing $\sigma_{\ell}$, the following result can be obtained.

Corollary 2.3 (see $[\mathbf{1 8}]$ ). Let $\sigma_{\ell}(t):=\left(1+e^{-t}\right)^{-1}, t \in \mathbf{R}$. For any given $f \in C^{0}[a, b]$, and for every $\varepsilon>0$, there exists $N \in \mathbf{N}^{+}$such that

$$
\left\|G_{N} f-f\right\|_{\infty}<\varepsilon
$$

where $G_{N} f$ is defined in (1) with $w>(N /(b-a)) \ln (N-1)$.

Corollary 2.3 provides an estimate for $w>0$, for every $N \in \mathbf{N}^{+}$, in the case of approximation of continuous functions by finite linear combination of logistic functions. 
Other interesting (and useful) examples of bounded sigmoidal functions are provided by the Gompertz functions, defined as

$$
\sigma_{\alpha \beta}(t):=e^{-\alpha e^{-\beta t}}, \quad t \in \mathbf{R},
$$

where the positive parameters $\alpha$ and $\beta$ represent an effective translation and a scaling, respectively. Gompertz functions find applications, e.g., in modeling tumor growth $[\mathbf{3}, \mathbf{1 7}, \mathbf{3 1}]$, and in population aging description [37]. Similarly to the case of logistic functions, the following can be established.

Corollary 2.4 (see $[\mathbf{1 8}]$ ). Let $\sigma_{\alpha \beta}(t):=e^{-\alpha e^{-\beta t}}, t \in \mathbf{R}, \alpha, \beta>0$. For any given $f \in C^{0}[a, b]$, and for every $\varepsilon>0$, there exists $N \in \mathbf{N}^{+}$ such that

$$
\left\|G_{N} f-f\right\|_{\infty}<\varepsilon
$$

where $G_{N} f$ is defined in (1) with

$$
w>\frac{N}{(b-a) \beta} \max \left\{\left|\ln \left(-\frac{1}{\alpha} \ln \left(\frac{N-1}{N}\right)\right)\right|,\left|\ln \left(\frac{1}{\alpha} \ln (N)\right)\right|\right\} .
$$

As in Corollary 2.3, Corollary 2.4 provides an estimate for the parameter $w>0$, when Gompertz sigmoidal functions are used.

Finally, we consider the special case of unit step (or Heaviside) sigmoidal functions, $H(t):=1$ for $t \geq 0$, and $H(t):=0$ for $t<0$. In this case, the results established in Theorem 2.1 hold true, and the neural networks of form (1) reduce to

$$
\begin{aligned}
\left(G_{N} f\right)(t):= & \sum_{k=1}^{N}\left[f\left(t_{k}\right)-f\left(t_{k-1}\right)\right] H\left(t-t_{k}\right) \\
& +f\left(t_{0}\right) H\left(t-t_{-1}\right),
\end{aligned}
$$

$t \in \mathbf{R}$, where $f \in C^{0}[a, b], h:=(b-a) / N$, and $t_{k}:=a+h k, k=$ $-1,0,1, \ldots, N,[\mathbf{1 8}, \mathbf{1 9}]$. Note that in $(2) G_{N} f$ becomes independent of the scaling parameter $w>0$.

Remark 2.5. Set $H_{k}(t):=H\left(t-t_{k}\right)$, with $H_{k}:[a, b] \rightarrow \mathbf{R}, t_{k}:=a+h k$, $h:=(b-a) / N$, for $k=-1,1, \ldots, N$, and

$$
\Sigma_{N}:=\operatorname{span}\left\{H_{k}: k=-1,1,2, \ldots, N\right\} .
$$


In [19], it was proved that the vector space $\Sigma_{N}$ has dimension $N+1$, and the set $\left\{H_{k}: k=-1,1,2, \ldots, N\right\}$ is a basis for it.

3. The collocation method. In this section, we introduce a collocation method aimed at solving nonlinear Volterra integrodifferential equations (VIDEs) of the neutral type.

In what follows, we consider initial value problems of the form

$$
\begin{gathered}
y^{\prime}(t)=f(t, y(t))+\int_{a}^{t} K\left(t, s, y(s), y^{\prime}(s)\right) d s, \\
y(a)=y_{0},
\end{gathered}
$$

for $t \in I:=[a, b]$, where $f: I \times \mathbf{R} \rightarrow \mathbf{R}$, and $K: \Omega \rightarrow \mathbf{R}$, since $\Omega:=I \times I \times \mathbf{R} \times \mathbf{R}$, are sufficiently smooth functions.

Suppose that (3) admits of a classical solution $y$, with $y \in C^{1}(I)$. Hence, $y^{\prime} \in C^{0}(I)$ and then, as a consequence of Theorem 2.1, $y(t)$ could be uniformly approximated on $I$ by a superposition of bounded sigmoidal functions. We choose to use unit step (sigmoidal) functions, and set

$$
\left(G_{N} y^{\prime}\right)(t):=\sum_{k=1}^{N} \alpha_{k} H\left(t-t_{k}\right)+\alpha_{0} H\left(t-t_{-1}\right), \quad t \in I
$$

with $G_{N} y^{\prime} \in \Sigma_{N}, N \in \mathbf{N}^{+}$. This will be a neural network which approximates $y^{\prime}$. Here, the coefficients $\alpha_{k}, k=0,1, \ldots, N$, are unknowns, and $t_{k}=a+k h, h=(b-a) / N$, for $k=-1,0, \ldots, N$. Integrating $G_{N} y^{\prime}$, we define

$$
\begin{aligned}
\left(S_{N} y\right)(t):= & \int_{a}^{t}\left(G_{N} y^{\prime}\right)(s) d s+y_{0} \\
= & \sum_{k=1}^{N} \alpha_{k} \int_{a}^{t} H\left(s-t_{k}\right) d s \\
& +\alpha_{0} \int_{a}^{t} H\left(s-t_{-1}\right) d s+y_{0},
\end{aligned}
$$

for $t \in I$, where $y_{0}$ is the initial data in (3). Clearly, $S_{N} y$ approximates $y$. Note that $G_{N} y^{\prime}$ and $S_{N} y$ are both characterized by the same 
unknown coefficients $\alpha_{k}$. By the definition of Heaviside functions, we have for every $t \in I$

$$
\int_{a}^{t} H\left(s-t_{-1}\right) d s=t-a, \quad \text { and } \quad \int_{a}^{t} H\left(s-t_{k}\right) d s=t-t_{k}
$$

for every $k$ such that $t_{k} \leq t$, and

$$
\int_{a}^{t} H\left(s-t_{k}\right) d s=0
$$

for every $k$ with $t_{k}>t, k=1, \ldots, N$. Then (5) becomes

$$
\left(S_{N} y\right)(t):=\sum_{k: t_{k} \leq t} \alpha_{k}\left(t-t_{k}\right)+y_{0}, \quad t \in I .
$$

Inserting $S_{N} y$ and $G_{N} y^{\prime}$ in (3), in place of $y$ and $y^{\prime}$, respectively, we obtain the collocation equation

$$
\begin{aligned}
\left(G_{N} y^{\prime}\right)(t)= & f\left(t,\left(S_{N} y\right)(t)\right) \\
& +\int_{a}^{t} K\left(t, s,\left(S_{N} y\right)(s),\left(G_{N} y^{\prime}\right)(s)\right) d s .
\end{aligned}
$$

If $\mathcal{C}_{N}:=\left\{t_{0}, t_{1}, \ldots, t_{N}\right\}$ denotes the set of the collocation points, we can evaluate $(7)$ at such points, obtaining

$$
\begin{aligned}
\left(G_{N} y^{\prime}\right)\left(t_{i}\right)= & f\left(t_{i},\left(S_{N} y\right)\left(t_{i}\right)\right) \\
& +\int_{a}^{t_{i}} K\left(t_{i}, s,\left(S_{N} y\right)(s),\left(G_{N} y^{\prime}\right)(s)\right) d s
\end{aligned}
$$

for every fixed $i, i=0,1, \ldots, N$. This is a algebraic system of $N+1$ equations where the unknowns are the $\alpha_{k}$ 's, $k=0,1, \ldots, N$. Solving such a system we obtain the $\alpha_{k}$ 's and then $S_{N} y$ and $G_{N} y^{\prime}$.

We will show that $S_{N} y$ and $G_{N} y^{\prime}$ do provide a simultaneous approximation of the solution, $y$, to (3) and of its first derivative, $y^{\prime}$. We can prove that system (8) always has a unique solution. Indeed, the following theorem holds.

Theorem 3.1. The algebraic system (8) has a unique solution $\alpha_{0}, \ldots, \alpha_{N}$, i.e., the collocation method based on unit step functions, 
used for solving the nonlinear integro-differential equations of the neutral type in (3), admits of a unique solution, $S_{N} y$, for every $N \in \mathbf{N}^{+}$. In particular, the coefficients $\alpha_{i}$ of $S_{N} y\left(\right.$ and $\left.G_{N} y^{\prime}\right)$, can be determined by the following explicit formula:

$$
\begin{gathered}
\alpha_{0}:=f\left(a, y_{0}\right), \\
\alpha_{1}:=f\left(t_{1}, \alpha_{0}\left(t_{1}-a\right)+y_{0}\right)-\alpha_{0} \\
+\int_{a}^{t_{1}} K\left(t_{1}, s, \alpha_{0}(s-a)+y_{0}, \alpha_{0}\right) d s,
\end{gathered}
$$

and

$$
\begin{aligned}
\alpha_{i}:= & f\left(t_{i}, \sum_{k=0}^{i-1} \alpha_{k}\left(t_{i}-t_{k}\right)+y_{0}\right)-\sum_{k=0}^{i-1} \alpha_{k} \\
& +\sum_{\nu=1}^{i} \int_{t_{\nu-1}}^{t_{\nu}} K\left(t_{i}, s, \sum_{k=0}^{\nu-1} \alpha_{k}\left(s-t_{k}\right)+y_{0}, \sum_{k=0}^{\nu-1} \alpha_{k}\right) d s
\end{aligned}
$$

for every $i, i=2, \ldots N$.

Proof. By equation (8), for $i=0,1, \ldots, N, N \in \mathbf{N}^{+}$, and the definition of $S_{N} y$ and $G_{N} y^{\prime}$, we have

$$
\begin{aligned}
& \sum_{k=1}^{N} \alpha_{k} H\left(t_{i}-t_{k}\right)+\alpha_{0} H\left(t_{i}-t_{-1}\right) \\
& =f\left(t_{i}, \sum_{k=0}^{i} \alpha_{k}\left(t_{i}-t_{k}\right)+y_{0}\right) \\
& \quad+\int_{a}^{t_{i}} K\left(t_{i}, s, \sum_{k=1}^{N} \alpha_{k} \int_{a}^{s} H\left(z-t_{k}\right) d z+\alpha_{0} \int_{a}^{s} H\left(z-t_{-1}\right) d z\right. \\
& \left.\quad+y_{0}, \sum_{k=1}^{N} \alpha_{k} H\left(s-t_{k}\right)+\alpha_{0} H\left(s-t_{-1}\right)\right) d s
\end{aligned}
$$


that can also be rewritten as

$$
\sum_{k=1}^{N} \alpha_{k} H\left(t_{i}-t_{k}\right)+\alpha_{0} H\left(t_{i}-t_{-1}\right)
$$$$
=f\left(t_{i}, \sum_{k=0}^{i} \alpha_{k}\left(t_{i}-t_{k}\right)+y_{0}\right)
$$

$$
\begin{aligned}
& +\sum_{\nu=1}^{i} \int_{t_{\nu-1}}^{t_{\nu}} K\left(t_{i}, s, \sum_{k=1}^{N} \alpha_{k} \int_{a}^{s} H\left(z-t_{k}\right) d z\right. \\
& \left.+\alpha_{0} \int_{a}^{s} H\left(z-t_{-1}\right) d z+y_{0}, \sum_{k=1}^{N} \alpha_{k} H\left(s-t_{k}\right)+\alpha_{0} H\left(s-t_{-1}\right)\right) d s .
\end{aligned}
$$

For $i=0$, equation (12) reduces to $\alpha_{0}=f\left(a, y_{0}\right)$. For $i=1$, (12) reduces to

$$
\begin{aligned}
\alpha_{0}+\alpha_{1}= & f\left(t_{1}, \alpha_{0}\left(t_{1}-a\right)+y_{0}\right) \\
& +\int_{a}^{t_{1}} K\left(t_{1}, s, \alpha_{0}(s-a)+y_{0}, \alpha_{0}\right) d s,
\end{aligned}
$$

and then we have

$$
\begin{aligned}
\alpha_{1}= & f\left(t_{1}, \alpha_{0}\left(t_{1}-a\right)+y_{0}\right)-\alpha_{0} \\
& +\int_{a}^{t_{1}} K\left(t_{1}, s, \alpha_{0}(s-a)+y_{0}, \alpha_{0}\right) d s,
\end{aligned}
$$

that can be immediately evaluated since $\alpha_{0}$ is known from the previous step. Note that, in general, for every fixed $i, i=2, \ldots, N, H\left(t_{i}-t_{k}\right)=$ 0 for every $k>i$, and $H\left(t_{i}-t_{k}\right)=1$ for every $k \leq i$. Moreover, it is easy to see that, for every $\nu=1, \ldots, i$, there is $H\left(\cdot-t_{k}\right)=1$ on $\left[t_{\nu-1}, t_{\nu}\right]$ for $k=0, \ldots, \nu-1$ and $H\left(\cdot-t_{k}\right)=0$ on $\left[t_{\nu-1}, t_{\nu}\right]$ for $k \geq \nu$. Finally note that, again for $\nu=1, \ldots, i$, if $s \in\left[t_{\nu-1}, t_{\nu}\right]$, we have

$$
\sum_{k=1}^{N} \alpha_{k} \int_{a}^{s} H\left(z-t_{k}\right) d z+\alpha_{0} \int_{a}^{s} H\left(z-t_{-1}\right) d z=\sum_{k=0}^{\nu-1} \alpha_{k}\left(s-t_{k}\right) .
$$

Thus, for the general case $i=2, \ldots, N,(12)$ becomes

$$
\begin{aligned}
\sum_{k=0}^{i} \alpha_{k}= & f\left(t_{i}, \sum_{k=0}^{i-1} \alpha_{k}\left(t_{i}-t_{k}\right)+y_{0}\right) \\
& +\sum_{\nu=1}^{i} \int_{t_{\nu-1}}^{t_{\nu}} K\left(t_{i}, s, \sum_{k=0}^{\nu-1} \alpha_{k}\left(s-t_{k}\right)+y_{0}, \sum_{k=0}^{\nu-1} \alpha_{k}\right) d s
\end{aligned}
$$


so we can conclude that

$$
\begin{aligned}
\alpha_{i}= & f\left(t_{i}, \sum_{k=0}^{i-1} \alpha_{k}\left(t_{i}-t_{k}\right)+y_{0}\right)-\sum_{k=0}^{i-1} \alpha_{k} \\
& +\sum_{\nu=1}^{i} \int_{t_{\nu-1}}^{t_{\nu}} K\left(t_{i}, s, \sum_{k=0}^{\nu-1} \alpha_{k}\left(s-t_{k}\right)+y_{0}, \sum_{k=0}^{\nu-1} \alpha_{k}\right) d s
\end{aligned}
$$

This shows that (8) admits of a unique solution, $\alpha_{0}, \ldots, \alpha_{N}$.

Note that, in Theorem 3.1, integrability of the kernel, $K$, in the integro-differential equation (3) is required. We emphasize the peculiarities of the present collocation method:

(i) one can determine all coefficients $\alpha_{k}$ in a very simple way,

(ii) a simultaneous approximation of $y$ and $y^{\prime}$ is provided by $S_{N} y$ and $G_{N} y^{\prime}$, respectively, as well as the analytical form of $S_{N} y$ and $G_{N} y^{\prime}$.

Remark 3.2. Note that, if the kernel in equation (3) is of the convolution type, i.e., $K\left(t, s, y(t), y^{\prime}(s)\right)=k(t-s) G\left(y(t), y^{\prime}(s)\right)$, and it does not depend on $y(s)$ (for all $s \in[0, t]$ ), then, formulae (10) and (11) in Theorem 3.1 reduce to

$$
\begin{aligned}
\alpha_{1}:= & f\left(t_{1}, \alpha_{0}\left(t_{1}-a\right)+y_{0}\right)-\alpha_{0}+G\left(\alpha_{0}\left(t_{1}-a\right)+y_{0}, \alpha_{0}\right) \\
& \times \int_{a}^{t_{1}} k\left(t_{1}-s\right) d s,
\end{aligned}
$$

and

$$
\begin{aligned}
\alpha_{i}:= & f\left(t_{i}, \sum_{k=0}^{i-1} \alpha_{k}\left(t_{i}-t_{k}\right)+y_{0}\right)-\sum_{k=0}^{i-1} \alpha_{k} \\
& +\sum_{\nu=1}^{i} G\left(\sum_{k=0}^{\nu-1} \alpha_{k}\left(t_{i}-t_{k}\right)+y_{0} \sum_{k=0}^{\nu-1} \alpha_{k}\right) \int_{t_{\nu-1}}^{t_{\nu}} k\left(t_{i}-s\right) d s,
\end{aligned}
$$

for every $i, i=2, \ldots N$. Now, changing the variable in the integrals, setting $z=s+h$ (where $h$ is the step-size of the collocation points), we obtain

$$
\int_{t_{\nu-1}}^{t_{\nu}} k\left(t_{i}-s\right) d s=\int_{t_{\nu-1}+h}^{t_{\nu}+h} k\left(t_{i}+h-z\right) d z=\int_{t_{\nu}}^{t_{\nu+1}} k\left(t_{i+1}-z\right) d z
$$


Relation (13) can be used to simplify the implementation of the collocation method in this case. In fact, in order to determine the coefficients $\alpha_{i}$, now we need to compute only one additional integral. In general we evaluate $i$ integrals at each step, while in the present approach we are able to reduce significantly the CPU time needed by our method.

The method can also be applied to other problems, such as classical nonlinear integro-differential equations of the form

$$
y^{\prime}(t)=f(t, y(t))+\int_{a}^{t} K(t, s, y(s)) d s, \quad y(0)=y_{0},
$$

for $t \in I:=[a, b]$, where $f: I \times \mathbf{R} \rightarrow \mathbf{R}$ and $K: I \times I \times \mathbf{R} \rightarrow \mathbf{R}$ are sufficiently smooth functions.

Moreover, a further class of VIDEs to which our collocation method can be applied is that of non-standard Volterra integro-differential equations having the typical general form

$$
y^{\prime}(t)=f(t, y(t))+\int_{a}^{t} K(t, s, y(t), y(s)) d s, \quad y(0)=y_{0},
$$

where $K: I \times I \times \mathbf{R} \times \mathbf{R} \rightarrow \mathbf{R}$ is sufficiently smooth and the integrand depends on both $y(s)$ and $y(t)$. The best known example of such a non-standard VIDE is given by the so-called logistic equation with memory term, see $[\mathbf{5 , ~ 6 , ~ 9 , ~ 2 2 , ~ 3 3 ] . ~ A s s u m e , ~ a s ~ a b o v e , ~ t h a t ~ ( 1 4 ) ~ o r ~}$ (15) admits of a classical solution. Proceeding as with neutral VIDEs, that is, replacing $y$ and $y^{\prime}$ with $S_{N} y$ and $G_{N} y^{\prime}$ in (14) or (15), and evaluating the ensuing collocation equations at the collocation points, we obtain algebraic systems similar to those in (8). The following theorems can be established by a straightforward adaptation of the proof of Theorem 3.1. For clarity, we state these results explicitly here.

Theorem 3.3. The collocation method based on unit step functions for solving the nonlinear VIDE (14), has a unique solution, $S_{N} y$, for every $N \in \mathbf{N}^{+}$. In particular, the coefficients $\alpha_{i}$ of $S_{N} y\left(\right.$ and $\left.G_{N} y^{\prime}\right)$, can be determined by the following explicit formula:

$$
\alpha_{0}:=f\left(a, y_{0}\right)
$$


(17)

$\alpha_{1}:=f\left(t_{1}, \alpha_{0}\left(t_{1}-a\right)+y_{0}\right)-\alpha_{0}+\int_{a}^{t_{1}} K\left(t_{1}, s, \alpha_{0}(s-a)+y_{0}\right) d s$

and

$$
\begin{aligned}
\alpha_{i}:= & f\left(t_{i}, \sum_{k=0}^{i-1} \alpha_{k}\left(t_{i}-t_{k}\right)+y_{0}\right)-\sum_{k=0}^{i-1} \alpha_{k} \\
& +\sum_{\nu=1}^{i} \int_{t_{\nu-1}}^{t_{\nu}} K\left(t_{i}, s, \sum_{k=0}^{\nu-1} \alpha_{k}\left(s-t_{k}\right)+y_{0}\right) d s,
\end{aligned}
$$

for every $i, i=2, \ldots N$.

Theorem 3.4. The collocation method based on unit step functions, for solving the non-standard VIDE (15), has a unique solution, $S_{N} y$, for every $N \in \mathbf{N}^{+}$. In particular, the coefficients $\alpha_{i}$ of $S_{N} y$ (and $\left.G_{N} y^{\prime}\right)$, can be determined by the following explicit formula:

$$
\begin{gathered}
\alpha_{0}:=f\left(a, y_{0}\right), \\
\alpha_{1}:=f\left(t_{1}, \alpha_{0}\left(t_{1}-a\right)+y_{0}\right)-\alpha_{0} \\
+\int_{a}^{t_{1}} K\left(t_{1}, s, \alpha_{0}\left(t_{1}-a\right)+y_{0}, \alpha_{0}(s-a)+y_{0}\right) d s,
\end{gathered}
$$

and

$$
\begin{aligned}
\alpha_{i}:= & f\left(t_{i}, \sum_{k=0}^{i-1} \alpha_{k}\left(t_{i}-t_{k}\right)+y_{0}\right)-\sum_{k=0}^{i-1} \alpha_{k} \\
& +\sum_{\nu=1}^{i} \int_{t_{\nu-1}}^{t_{\nu}} K\left(t_{i}, s, \sum_{k=0}^{i-1} \alpha_{k}\left(t_{i}-t_{k}\right)+y_{0}, \sum_{k=0}^{\nu-1} \alpha_{k}\left(s-t_{k}\right)+y_{0}\right) d s
\end{aligned}
$$

for every $i, i=2, \ldots N$.

Remark 3.5. Suppose that $y \in C^{1}(I)$ is the classical solution of a VIDE of the form in (3), and that $S_{N} y$ is its collocation solution, written with coefficients $\alpha_{k}$ provided by Theorem 3.1. Moreover, $G_{N} y^{\prime}$ is given in terms of unit step functions, and this represents an 
approximation of $y^{\prime}$. Now let $\sigma$ be a fixed bounded sigmoidal function. By Remark 2.2, we know that various approximations of any given continuous function can be obtained using various different sigmoidal functions, still retaining the same coefficients. Therefore, using the same $\alpha_{k}$, we can define

$$
\left(G_{N}^{\sigma} y^{\prime}\right)(t):=\sum_{k=1}^{N} \alpha_{k} \sigma\left(w\left(t-t_{k}\right)\right)+\alpha_{0} \sigma\left(w\left(t-t_{-1}\right)\right), \quad t \in I,
$$

where $w$ is a suitable positive parameter depending on $\sigma$ and $N$, and the $t_{k}$ 's are the uniformly spaced nodes in the interval $[a, b]$ defined above. It follows that $G_{N}^{\sigma} y^{\prime}$ provides a further approximation to $y^{\prime}$, and hence we obtain, by integration,

$$
\begin{aligned}
\left(S_{N}^{\sigma} y\right)(t):= & \sum_{k=1}^{N} \alpha_{k} \int_{a}^{t} \sigma\left(w\left(s-t_{k}\right)\right) d s \\
& +\alpha_{0} \int_{a}^{t} \sigma\left(w\left(s-t_{-1}\right)\right) d s+y_{0}, \quad t \in I,
\end{aligned}
$$

which provides a further approximation to $y$ on $I$, for every $N \in \mathbf{N}^{+}$.

The same observation can be made when our collocation method is applied to VIDEs of the form (14) or (15), using the same coefficients $\alpha_{k}$ determined in Theorem 3.3 and Theorem 3.4, respectively.

4. Error analysis. In this section, we analyze the various sources of numerical errors which affect our collocation method.

4.1. A priori estimates. We start by considering our collocation method with unit step functions, applying it to the VIDEs of neutral type in (3).

First of all, we define the error function for $y^{\prime}$,

$$
e_{N}(t):=y^{\prime}(t)-\left(G_{N} y^{\prime}\right)(t), \quad t \in I:=[a, b],
$$

where $y$ is the classical solution of (3) and $G_{N} y^{\prime}$ is the neural network given in (4) and written with coefficients $\alpha_{k}$ determined by our collocation method for (3). Integrating $e_{N}$, we obtain

$$
E_{N}(t):=\int_{a}^{t} e_{N}(z) d z=y(t)-\left(S_{N} y\right)(t), \quad t \in I:=[a, b],
$$


that is, the error function for $y$. The following theorem provides some a priori estimates for $e_{N}$ and $E_{N}$.

Theorem 4.1. Let (3) be a given VIDE of the neutral type, which admits of a classical solution $y \in C^{1}(I)$. Assume that there exist the positive constants $L_{f}, C, L_{1}$ and $L_{2}$, such that

(i) for every $\left(t_{1}, s_{1}\right),\left(t_{2}, s_{2}\right) \in I \times \mathbf{R}$,

$$
\left|f\left(t_{1}, s_{1}\right)-f\left(t_{2}, s_{2}\right)\right| \leq L_{f}\left\|\left(t_{1}, s_{1}\right)-\left(t_{2}, s_{2}\right)\right\|_{2},
$$

where $\|\cdot\|_{2}$ is the Euclidean norm in $\mathbf{R}^{2}$;

(ii) for every $(t, s, x, z) \in I \times I \times\left[-\|y\|_{\infty},+\|y\|_{\infty}\right] \times\left[-\left\|y^{\prime}\right\|_{\infty},+\left\|y^{\prime}\right\|_{\infty}\right]$, we have $|K(t, s, x, z)| \leq C$;

(iii) for every $\left(t_{1}, s, x, z\right),\left(t_{2}, s, x, z\right) \in I \times I \times\left[-\|y\|_{\infty},\|y\|_{\infty}\right] \times$ $\left[-\left\|y^{\prime}\right\|_{\infty},\left\|y^{\prime}\right\| \infty\right]$

$$
\left|K\left(t_{1}, s, x, z\right)-K\left(t_{2}, s, x, z\right)\right| \leq L_{1}\left|t_{1}-t_{2}\right|
$$

(iv) for every $\left(t, s, x_{1}, z_{1}\right),\left(t, s, x_{2}, z_{2}\right) \in I \times I \times \mathbf{R} \times \mathbf{R}$,

$$
\left|K\left(t, s, x_{1}, z_{1}\right)-K\left(t, s, x_{2}, z_{2}\right)\right| \leq L_{2}\left\|\left(x_{1}, z_{1}\right)-\left(x_{2}, z_{2}\right)\right\|_{2} .
$$

Then,

$$
\left|e_{N}(t)\right| \leq \frac{(b-a) M_{1}}{N} e^{M_{2}(t-a)}, \quad t \in I:=[a, b],
$$

and

$$
\left|E_{N}(t)\right| \leq \frac{(b-a) M_{1}}{N M_{2}}\left(e^{M_{2}(t-a)}-1\right), \quad t \in I,
$$

for every $N \in \mathbf{N}^{+}$, where $M_{1}$ and $M_{2}$ are suitable positive constants not dependent on $N$.

Proof. Let $N \in \mathbf{N}^{+}$and $t \in I:=[a, b]$ be fixed. Define

$$
j:=\max \left\{i: t_{i} \leq t, t_{i} \in \mathcal{C}_{N}, \quad i=0,1, \ldots, N\right\}
$$


where $t_{i}=a+k h, h:=(b-a) / N, k=0,1, \ldots, N$, are the collocation points. We can write

$$
\begin{aligned}
\left|e_{N}(t)\right| \leq & \left|e_{N}(t)-e_{N}\left(t_{j}\right)\right|+\left|e_{N}\left(t_{j}\right)\right| \leq\left|y^{\prime}(t)-y^{\prime}\left(t_{j}\right)\right| \\
& +\left|\left(G_{N} y^{\prime}\right)(t)-\left(G_{N} y^{\prime}\right)\left(t_{j}\right)\right|+\left|e_{N}\left(t_{j}\right)\right| \\
= & \left|y^{\prime}(t)-y^{\prime}\left(t_{j}\right)\right|+\left|e_{N}\left(t_{j}\right)\right|,
\end{aligned}
$$

because $\left(G_{N} y^{\prime}\right)(t)=\left(G_{N} y^{\prime}\right)\left(t_{j}\right)$, since the function $G_{N} y^{\prime}$ is written as a superposition of unit step functions and hence it is piecewise constant. Now,

$$
\begin{aligned}
\left|e_{N}(t)\right| \leq & \left|y^{\prime}(t)-y^{\prime}\left(t_{j}\right)\right|+\left|e_{N}\left(t_{j}\right)\right| \\
= & \mid f(t, y(t))+\int_{a}^{t} K\left(t, s, y(s), y^{\prime}(s)\right) d s \\
& -f\left(t_{j}, y\left(t_{j}\right)\right)-\int_{a}^{t_{j}} K\left(t_{j}, s, y(s), y^{\prime}(s)\right) d s \mid \\
& +\mid f\left(t_{j}, y\left(t_{j}\right)\right)+\int_{a}^{t_{j}}\left[K\left(t_{j}, s, y(s), y^{\prime}(s)\right)\right. \\
& \left.-K\left(t_{j}, s,\left(S_{N} y\right)(s),\left(G_{N} y^{\prime}\right)(s)\right)\right] d s \\
& -f\left(t_{j},\left(S_{N} y\right)\left(t_{j}\right)\right)|\leq| f(t, y(t))-f\left(t_{j}, y\left(t_{j}\right)\right) \mid \\
& +\int_{t_{j}}^{t}\left|K\left(t, s, y(s), y^{\prime}(s)\right)\right| d s \\
& +\int_{a}^{t_{j}}\left|K\left(t, s, y(s), y^{\prime}(s)\right)-K\left(t_{j}, s, y(s), y^{\prime}(s)\right)\right| d s \\
& +\left|f\left(t_{j}, y\left(t_{j}\right)\right)-f\left(t_{j},\left(S_{N} y\right)\left(t_{j}\right)\right)\right| \\
& +\int_{a}^{t_{j}}\left|K\left(t_{j}, s, y(s), y^{\prime}(s)\right)-K\left(t_{j}, s,\left(S_{N} y\right)(s), y^{\prime}(s)\right)\right| d s \\
& +\int_{a}^{t_{j}} \mid K\left(t_{j}, s,\left(S_{N} y\right)(s), y^{\prime}(s)\right) \\
& -K\left(t_{j}, s,\left(S_{N} y\right)(s),\left(G_{N} y^{\prime}\right)(s)\right) \mid d s \\
= & J_{1}+J_{2}+J_{3}+J_{4}+J_{5}+J_{6} .
\end{aligned}
$$

We first estimate $J_{1}$ and $J_{4}$. Being $y \in C^{1}(I), y$ is Lipschitz continuous on $I$, with some Lipschitz constant, say, $L_{y}>0$. Thus, we obtain from 
condition (i)

$$
J_{1} \leq L_{f} \sqrt{\left(t-t_{j}\right)^{2}+\left(y(t)-y\left(t_{j}\right)\right)^{2}} \leq L_{f} \sqrt{h^{2}\left(1+L_{y}^{2}\right)}=: h M
$$

and, moreover,

$$
J_{4} \leq L_{f}\left|y\left(t_{j}\right)-\left(S_{N} y\right)\left(t_{j}\right)\right|=L_{f}\left|E_{N}\left(t_{j}\right)\right| \leq L_{f} \int_{a}^{t_{j}}\left|e_{N}(z)\right| d z .
$$

As, for $J_{2}$, we can easily infer from condition (ii) that $J_{2} \leq C\left(t-t_{j}\right) \leq$ $C h$. Turning our attention to $J_{3}$, we have by condition (iii)

$$
J_{3} \leq L_{1}\left|t-t_{j}\right|\left(t_{j}-a\right) \leq L_{1}\left(t_{j}-a\right) h \leq L_{1}(b-a) h .
$$

We finally estimate $J_{5}$ and $J_{6}$. From condition (iv), we have

$$
J_{5} \leq L_{2} \int_{a}^{t_{j}}\left|y(s)-\left(S_{N} y\right)(s)\right| d s=L_{2} \int_{a}^{t_{j}}\left|E_{N}(s)\right| d s,
$$

and similarly

$$
J_{6} \leq L_{2} \int_{a}^{t_{j}}\left|y^{\prime}(s)-\left(G_{N} y^{\prime}\right)(s)\right| d s=L_{2} \int_{a}^{t_{j}}\left|e_{N}(s)\right| d s .
$$

Therefore, combining all such estimates we conclude that

$$
\begin{aligned}
\left|e_{N}(t)\right| \leq & h\left[M+C+L_{1}(b-a)\right] \\
& +L_{2} \int_{a}^{t_{j}}\left|E_{N}(s)\right| d s+\left(L_{f}+L_{2}\right) \int_{a}^{t_{j}}\left|e_{N}(z)\right| d z \\
= & h\left[M+C+L_{1}(b-a)\right]+L_{2} \int_{a}^{t_{j}}\left|\int_{a}^{s} e_{N}(z) d z\right| d s \\
& +\left(L_{f}+L_{2}\right) \int_{a}^{t_{j}}\left|e_{N}(z)\right| d z \\
\leq & h\left[M+C+L_{1}(b-a)\right]+L_{2} \int_{a}^{t_{j}}\left[\int_{a}^{t_{j}}\left|e_{N}(z)\right| d z\right] d s \\
& +\left(L_{f}+L_{2}\right) \int_{a}^{t_{j}}\left|e_{N}(z)\right| d z
\end{aligned}
$$




$$
\begin{aligned}
\leq & h\left[M+C+L_{1}(b-a)\right] \\
& +\left[L_{2}(b-a+1)+L_{f}\right] \int_{a}^{t_{j}}\left|e_{N}(z)\right| d z \\
\leq & h\left[M+C+L_{1}(b-a)\right] \\
& +\left[L_{2}(b-a+1)+L_{f}\right] \int_{a}^{t}\left|e_{N}(z)\right| d z \\
= & h M_{1}+M_{2} \int_{a}^{t}\left|e_{N}(z)\right| d z .
\end{aligned}
$$

The previous inequality holds for every $t \in I$, and thus we obtain, by Gronwall's lemma,

$$
\left|e_{N}(t)\right| \leq h M_{1} e^{M_{2}(t-a)}=\frac{(b-a) M_{1}}{N} e^{M_{2}(t-a)}, \quad t \in I,
$$

for every $N \in \mathbf{N}^{+}$. Moreover,

$$
\begin{aligned}
\left|E_{N}(t)\right| & \leq \int_{a}^{t}\left|e_{N}(s)\right| d s \leq \frac{(b-a) M_{1}}{N} \int_{a}^{t} e^{M_{2}(s-a)} d s \\
& =\frac{(b-a) M_{1}}{N M_{2}}\left(e^{M_{2}(t-a)}-1\right)
\end{aligned}
$$

for every $N \in \mathbf{N}^{+}$.

Remark 4.2. We can infer from Theorem 4.1 that

$$
\left\|e_{N}\right\|_{\infty} \leq \frac{(b-a) M_{1}}{N} e^{M_{2}(b-a)}
$$

and

$$
\left\|E_{N}\right\|_{\infty} \leq \frac{(b-a) M_{1}}{N M_{2}}\left(e^{M_{2}(b-a)}-1\right),
$$

so that we can conclude that $\left\|e_{N}\right\|_{\infty} \rightarrow 0$ and $\left\|E_{N}\right\|_{\infty} \rightarrow 0$ as $N \rightarrow+\infty: S_{N} y$ and $G_{N} y^{\prime}$ converge both uniformly on $I$, to $y$ and $y^{\prime}$, respectively.

Remark 4.3. In Theorem 4.1, the Lipschitz conditions (i) and (iv) are global. This is a technical assumption, which circumvents the need of 
having an a priori boundedness of $S_{N} y$ and $G_{N} y^{\prime}$. For such a reason, Theorem 4.1 cannot cover, e.g., the case of VIDEs like (3) with kernels of the form $K(t, s, x, z)=\widetilde{K}(t, s) x^{p} z^{p}$, or $f(t, s)=\widetilde{f}(t) s^{p}$, with $p>1$, see also $[\mathbf{1 9}]$.

Theorem 4.1 can also be extended in such a way to apply our collocation method to (14). Clearly, the coefficients $\alpha_{k}$ of $S_{N} y$ (and $G_{N} y^{\prime}$ ) in (14) are the same obtained in Theorem 3.3 (or in Theorem 3.4, in case of the equations of the kind in (15)). The following can be proved.

Theorem 4.4. Let a VIDE as in (14) be given, which admits a classical solution $y \in C^{1}(I)$. Assume that there exist positive constants $L_{f}, C, L_{1}$ and $L_{2}$, such that:

(i) for every $\left(t_{1}, s_{1}\right),\left(t_{2}, s_{2}\right) \in I \times \mathbf{R}$,

$$
\left|f\left(t_{1}, s_{1}\right)-f\left(t_{2}, s_{2}\right)\right| \leq L_{f}\left\|\left(t_{1}, s_{1}\right)-\left(t_{2}, s_{2}\right)\right\|_{2} ;
$$

(ii) for every $(t, s, x) \in I \times I \times\left[-\|y\|_{\infty},+\|y\|_{\infty}\right]$, we have $|K(t, s, x)| \leq$ $C$;

(iii) for every $\left(t_{1}, s, x\right),\left(t_{2}, s, x\right) \in I \times I \times\left[-\|y\|_{\infty},\|y\|_{\infty}\right]$,

$$
\left|K\left(t_{1}, s, x\right)-K\left(t_{2}, s, x\right)\right| \leq L_{1}\left|t_{1}-t_{2}\right| ;
$$

(iv) for every $\left(t, s, x_{1}\right),\left(t, s, x_{2}\right) \in I \times I \times \mathbf{R}$,

$$
\left|K\left(t, s, x_{1}\right)-K\left(t, s, x_{2}\right)\right| \leq L_{2}\left|x_{1}-x_{2}\right| .
$$

Then,

$$
\left|e_{N}(t)\right| \leq \frac{(b-a) M_{1}}{N} e^{M_{2}(t-a)}, \quad t \in I:=[a, b],
$$

and

$$
\left|E_{N}(t)\right| \leq \frac{(b-a) M_{1}}{N M_{2}}\left(e^{M_{2}(t-a)}-1\right), \quad t \in I,
$$

for every $N \in \mathbf{N}^{+}$, where $M_{1}$ and $M_{2}$ are suitable positive constants not depending on $N$. 
The proof of this theorem is similar to that of Theorem 4.1, and thus is omitted. Moreover, in case of non-standard VIDEs like those in (15), we have the following.

Theorem 4.5. Let there be a fixed non-standard VIDE like that in (15), which admits a classical solution $y \in C^{1}(I)$. Assume that there exist positive constants $L_{f}, C, L_{1}$ and $L_{2}$, such that

(i) for every $\left(t_{1}, s_{1}\right),\left(t_{2}, s_{2}\right) \in I \times \mathbf{R}$,

$$
\left|f\left(t_{1}, s_{1}\right)-f\left(t_{2}, s_{2}\right)\right| \leq L_{f}\left\|\left(t_{1}, s_{1}\right)-\left(t_{2}, s_{2}\right)\right\|_{2}
$$

(ii) for every $(t, s, x, z) \in I \times I \times\left[-\|y\|_{\infty},+\|y\|_{\infty}\right] \times\left[-\|y\|_{\infty},+\|y\|_{\infty}\right]$, we have $|K(t, s, x, z)| \leq C$;

(iii) for every $\left(t_{1}, s, x, z\right),\left(t_{2}, s, x, z\right) \in I \times I \times\left[-\|y\|_{\infty},\|y\|_{\infty}\right] \times$ $\left[-\|y\|_{\infty},\|y\|_{\infty}\right]$

$$
\left|K\left(t_{1}, s, x, z\right)-K\left(t_{2}, s, x, z\right)\right| \leq L_{1}\left|t_{1}-t_{2}\right|
$$

(iv) for every $\left(t, s, x_{1}, z_{1}\right)$, $\left(t, s, x_{2}, z_{2}\right) \in I \times I \times \mathbf{R} \times \mathbf{R}$,

$$
\left|K\left(t, s, x_{1}, z_{1}\right)-K\left(t, s, x_{2}, z_{2}\right)\right| \leq L_{2}\left\|\left(x_{1}, z_{1}\right)-\left(x_{2}, z_{2}\right)\right\|_{2} .
$$

Then,

$$
\left|e_{N}(t)\right| \leq \frac{(b-a) M_{1}}{N} e^{M_{2}(t-a)}, \quad t \in I:=[a, b]
$$

and

$$
\left|E_{N}(t)\right| \leq \frac{(b-a) M_{1}}{N M_{2}}\left(e^{M_{2}(t-a)}-1\right), \quad t \in I
$$

for every $N \in \mathbf{N}^{+}$, where $M_{1}$ and $M_{2}$ are suitable positive constants not depending on $N$.

Proof. As in the proof of Theorem 4.1 (and with the same notation), we can write, for every fixed $t \in I$, 


$$
\begin{aligned}
\left|e_{N}(t)\right| \leq & \left|y^{\prime}(t)-y^{\prime}\left(t_{j}\right)\right|+\left|e_{N}\left(t_{j}\right)\right| \\
= & \mid f(t, y(t))+\int_{a}^{t} K(t, s, y(t), y(s)) d s-f\left(t_{j}, y\left(t_{j}\right)\right) \\
& -\int_{a}^{t_{j}} K\left(t_{j}, s, y\left(t_{j}\right), y(s)\right) d s \mid \\
& +\mid f\left(t_{j}, y\left(t_{j}\right)\right)+\int_{a}^{t_{j}}\left[K\left(t_{j}, s, y\left(t_{j}\right), y(s)\right)\right. \\
& \left.-K\left(t_{j}, s,\left(S_{N} y\right)\left(t_{j}\right),\left(S_{N} y\right)(s)\right)\right] d s-f\left(t_{j},\left(S_{N} y\right)\left(t_{j}\right)\right) \mid \\
\leq & \left|f(t, y(t))-f\left(t_{j}, y\left(t_{j}\right)\right)\right| \\
& +\int_{t_{j}}^{t}|K(t, s, y(t), y(s))| d s \\
& +\int_{a}^{t_{j}}\left|K(t, s, y(t), y(s))-K\left(t_{j}, s, y\left(t_{j}\right), y(s)\right)\right| d s \\
& +\left|f\left(t_{j}, y\left(t_{j}\right)\right)-f\left(t_{j},\left(S_{N} y\right)\left(t_{j}\right)\right)\right| \\
& +\int_{a}^{t_{j}} \mid K\left(t_{j}, s, y\left(t_{j}\right), y(s)\right) \\
& -K\left(t_{j}, s,\left(S_{N} y\right)\left(t_{j}\right), y(s)\right) \mid d s \\
& +\int_{a}^{t_{j}} \mid K\left(t_{j}, s,\left(S_{N} y\right)\left(t_{j}\right), y(s)\right) \\
& -K_{1}\left(t_{j}, s,\left(S_{N} y\right)\left(t_{j}\right),\left(S_{N} y\right)(s)\right) \mid d s \\
& J_{1}+J_{3}+J_{4}+J_{5}+J_{6} .
\end{aligned}
$$

The terms $J_{1}, J_{2}$ and $J_{4}$ can be estimated as in Theorem 4.1. As for $J_{3}, J_{5}$ and $J_{6}$, we obtain, exploiting (iii) and (iv) and the fact that $y$ is Lipschitz continuous with Lipschitz constant $L_{y}>0$,

$$
\begin{aligned}
J_{3} & \leq L_{1}\left(t_{j}-a\right) \sqrt{\left(t-t_{j}\right)^{2}+\left(y(t)-y\left(t_{j}\right)\right)^{2}} \\
& \leq L_{1}\left(t_{j}-a\right) \sqrt{h^{2}\left(1+L_{y}^{2}\right)} \\
& \leq L_{1}(b-a) \sqrt{\left(1+L_{y}^{2}\right)} h
\end{aligned}
$$


and

$J_{5} \leq L_{2}\left|E_{N}\left(t_{j}\right)\right| \int_{a}^{t_{j}} d s \leq L_{2}(b-a)\left|E_{N}\left(t_{j}\right)\right| \leq L_{2}(b-a) \int_{a}^{t}\left|e_{N}(z)\right| d z$,

and finally,

$$
\begin{aligned}
J_{6} & \leq L_{2} \int_{a}^{t_{j}}\left|E_{N}(s)\right| d s \leq L_{2}(b-a) \int_{a}^{t_{j}} \int_{a}^{s}\left|e_{N}(z)\right| d z d s \\
& \leq L_{2}(b-a)^{2} \int_{a}^{t_{j}}\left|e_{N}(z)\right| d z \\
& \leq L_{2}(b-a)^{2} \int_{a}^{t}\left|e_{N}(z)\right| d z .
\end{aligned}
$$

The proof then follows as in Theorem 4.1.

4.2. A posteriori estimates. As noted in Remark 4.3, the a priori estimates made in subsection 4.1 hold only for equations with kernels $K$ and data $f$ which are globally Lipschitz. However, if we replace conditions (i) and (iv) in Theorem 4.1 with a local Lipschitz condition, some a posteriori error estimates can be established. Indeed, the following can be proved.

Theorem 4.6. Let a given VIDE of neutral type like that in (3) have a classical solution $y \in C^{1}(I)$, and assume that conditions (ii) and (iii) of Theorem 4.1 hold for some positive constants $C$ and $L_{1}$, respectively. Suppose in addition that:

(a) there exist a constant $L_{f}>0$ and a function $\mathcal{L}_{f}: \mathbf{R}_{0}^{+} \rightarrow \mathbf{R}_{0}^{+}$, such that, for every $\gamma>0$,

$$
\left|f\left(t_{1}, s_{1}\right)-f\left(t_{2}, s_{2}\right)\right| \leq \mathcal{L}_{f}(\gamma)\left|f\left(t_{1}, s_{1} / \gamma\right)-f\left(t_{2}, s_{2} / \gamma\right)\right|
$$

for every $\left(t_{1}, s_{1}\right),\left(t_{2}, s_{2}\right) \in I \times \mathbf{R}$, and such that

$$
\left|f\left(t_{1}, s_{1}\right)-f\left(t_{2}, s_{2}\right)\right| \leq L_{f}\left\|\left(t_{1}, s_{1}\right)-\left(t_{2}, s_{2}\right)\right\|_{2}
$$

for every $\left(t_{1}, s_{1}\right),\left(t_{2}, s_{2}\right) \in I \times[-1,1]$; 
(b) there exist a constant $L_{2}>0$ and a function $\mathcal{L}_{K}: \mathbf{R}_{0}^{+} \rightarrow \mathbf{R}_{0}^{+}$ such that, for every constant $\gamma>0$,

$$
\begin{aligned}
& \left|K\left(t_{1}, s_{1}, x_{1}, z_{1}\right)-K\left(t_{2}, s_{2}, x_{2}, z_{2}\right)\right| \\
& \quad \leq \mathcal{L}_{K}(\gamma)\left|K\left(t_{1}, s_{1}, x_{1} / \gamma, z_{1} / \gamma\right)-K\left(t_{2}, s_{2}, x_{2} / \gamma, z_{2} / \gamma\right)\right|
\end{aligned}
$$

for every $\left(t_{1}, s_{1}, x_{1}, z_{1}\right),\left(t_{2}, s_{2}, x_{2}, z_{2}\right) \in I \times I \times \mathbf{R} \times \mathbf{R}$, and such that

$$
\left|K\left(t, s, x_{1}, z_{1}\right)-K\left(t, s, x_{2}, z_{2}\right)\right| \leq L_{2}\left\|\left(x_{1}, z_{1}\right)-\left(x_{2}, z_{2}\right)\right\|_{2}
$$

for every $\left(t, s, x_{1}, z_{1}\right),\left(t, s, x_{2}, z_{2}\right) \in I \times I \times[-1,1] \times[-1,1]$. Then,

$$
\left|e_{N}(t)\right| \leq \frac{(b-a) M_{1}}{N} e^{\mathcal{M}_{N}(t-a)}, \quad t \in I=[a, b]
$$

and

$$
\left|E_{N}(t)\right| \leq \frac{(b-a) M_{1}}{N \mathcal{M}_{N}}\left(e^{\mathcal{M}_{N}(t-a)}-1\right), \quad t \in I
$$

where

$$
\mathcal{M}_{N}:=\frac{M_{2}}{\gamma_{N}}\left\{\mathcal{L}_{f}\left(\gamma_{N}\right)+\mathcal{L}_{K}\left(\gamma_{N}\right)\right\}
$$

$M_{1}$ and $M_{2}$ being suitable positive constants not depending on $N$, and

$$
\gamma_{N}:=\max \left\{\|y\|_{\infty},\left\|y^{\prime}\right\|_{\infty},\left\|S_{N} y\right\|_{\infty},\left\|G_{N} y^{\prime}\right\|_{\infty}\right\}
$$

for every $N \in \mathbf{N}^{+}$.

Proof. We define $J_{i}, i=1, \ldots, 6$ as in the proof of Theorem 4.1, for $t \in I$ fixed. We first estimate $J_{1}, J_{2}$ and $J_{3}$, as in the proof of Theorem 4.1. Now, we estimate $J_{4}, J_{5}$ and $J_{6}$. Let $\gamma_{N}>0$ be the constant defined in (26). Using condition (a), we obtain

$$
\begin{aligned}
J_{4} & \leq \mathcal{L}_{f}\left(\gamma_{N}\right)\left|f\left(t_{j}, y\left(t_{j}\right) / \gamma_{N}\right)-f\left(t_{j},\left(S_{N} y\right)\left(t_{j}\right) / \gamma_{N}\right)\right| \\
& \leq L_{f} \frac{\mathcal{L}_{f}\left(\gamma_{N}\right)}{\gamma_{N}}\left|E_{N}\left(t_{j}\right)\right| \leq L_{f} \frac{\mathcal{L}_{f}\left(\gamma_{N}\right)}{\gamma_{N}} \int_{a}^{t}\left|e_{N}(s)\right| d s
\end{aligned}
$$


with $\left|y\left(t_{j}\right)\right| / \gamma_{N},\left|\left(S_{N} y\right)\left(t_{j}\right)\right| / \gamma_{N} \leq 1$. Furthermore, we have from condition (b),

$$
\begin{aligned}
J_{5} & \leq \mathcal{L}_{K}\left(\gamma_{N}\right) \int_{a}^{t_{j}}\left|K\left(t_{j}, s, \frac{y(s)}{\gamma_{N}}, \frac{y^{\prime}(s)}{\gamma_{N}}\right)-K\left(t_{j}, s, \frac{\left(S_{N} y\right)(s)}{\gamma_{N}}, \frac{y^{\prime}(s)}{\gamma_{N}}\right)\right| d s \\
& \leq L_{2} \mathcal{L}_{K}\left(\gamma_{N}\right) \int_{a}^{t_{j}}\left|y(s) / \gamma_{N}-\left(S_{N} y\right)(s) / \gamma_{N}\right| d s \\
& =L_{2} \frac{\mathcal{L}_{K}\left(\gamma_{N}\right)}{\gamma_{N}} \int_{a}^{t_{j}}\left|E_{N}(s)\right| d s \\
& \leq L_{2} \frac{\mathcal{L}_{K}\left(\gamma_{N}\right)}{\gamma_{N}} \int_{a}^{t_{j}} \int_{a}^{s}\left|e_{N}(z)\right| d z d s \leq L_{2}(b-a) \frac{\mathcal{L}_{K}\left(\gamma_{N}\right)}{\gamma_{N}} \int_{a}^{t}\left|e_{N}(z)\right| d z .
\end{aligned}
$$

Similarly,

$$
J_{6} \leq L_{2} \frac{\mathcal{L}_{K}\left(\gamma_{N}\right)}{\gamma_{N}} \int_{a}^{t_{j}}\left|e_{N}(s)\right| d s \leq L_{2} \frac{\mathcal{L}_{K}\left(\gamma_{N}\right)}{\gamma_{N}} \int_{a}^{t}\left|e_{N}(s)\right| d s .
$$

Proceeding as in Theorem 4.1,

$$
\left|e_{N}(t)\right| \leq h M_{1}+M_{2}\left\{\frac{\mathcal{L}_{f}\left(\gamma_{N}\right)}{\gamma_{N}}+\frac{\mathcal{L}_{K}\left(\gamma_{N}\right)}{\gamma_{N}}\right\} \int_{a}^{t}\left|e_{N}(z)\right| d z,
$$

for every $t \in I=[a, b]$, with $h=(b-a) / N$, the constant $M_{1}$ being defined as in Theorem 4.1 and $M_{2}:=2 \max \left\{L_{f}, L_{2}(b-a), L_{2}\right\}$. Then, we obtain from Gronwall's lemma

$$
\left|e_{N}(t)\right| \leq \frac{(b-a) M_{1}}{N} e^{\mathcal{M}_{N}(t-a)}, \quad t \in I=[a, b],
$$

where

$$
\mathcal{M}_{N}:=\frac{M_{2}}{\gamma_{N}}\left\{\mathcal{L}_{f}\left(\gamma_{N}\right)+\mathcal{L}_{K}\left(\gamma_{N}\right)\right\}
$$

and moreover,

$$
\left|E_{N}(t)\right| \leq \frac{(b-a) M_{1}}{N \mathcal{M}_{N}}\left(e^{\mathcal{M}_{N}(t-a)}-1\right), \quad t \in I,
$$

for every $N \in \mathbf{N}^{+}$. 
Note that all examples of functions $f$ and kernels $K$ mentioned in Remark 4.3 do satisfy conditions (a) and (b) of Theorem 4.6.

Remark 4.7 . Theorem 4.6 can be easily extended to provide estimates for the error affecting our method for VIDEs like (14) and (15), as in Theorems 4.4 and 4.5 .

We can now use the estimate provided by Theorem 4.1 (or Theorem 4.6) to derive other estimates for the errors affecting the variant of our method introduced in Remark 3.5 for equations like (3).

Let $\sigma$ be a fixed bounded sigmoidal function. In what follows, we denote by

$$
e_{N}^{\sigma}(t):=y^{\prime}(t)-\left(G_{N}^{\sigma} y^{\prime}\right)(t), \quad t \in I:=[a, b],
$$

where $G_{N}^{\sigma} y^{\prime}$ is defined in (22) and it is written with the coefficients $\alpha_{k}$ determined by our collocation method for equation (3). Furthermore, we denote by

$$
E_{N}^{\sigma}(t):=\int_{a}^{t} e_{N}^{\sigma}(z) d z=y(t)-\left(S_{N}^{\sigma} y\right)(t), \quad t \in I:=[a, b]
$$

that is, the error function for $y$, when the approximate solution is expressed by $S_{N}^{\sigma} y$ defined in (23). The following theorem provides some a posteriori estimates for the error functions $e_{N}^{\sigma}$ and $E_{N}^{\sigma}$.

Theorem 4.8. Let $\sigma$ be a bounded sigmoidal function, and let (3) be a given VIDE of the neutral type, which admits of a classical solution, $y \in C^{1}(I)$. Under the assumptions (i)-(iv) of Theorem 4.1, we have

$$
\begin{aligned}
\left|e_{N}^{\sigma}(t)\right| \leq & \frac{(b-a) M_{1}}{N} e^{M_{2}(t-a)} \\
& +\sum_{k: t_{k} \leq t}\left|\alpha_{k}\right|\left|\sigma\left(w\left(t-t_{k}\right)\right)-1\right| \\
& +\sum_{k: t_{k}>t}\left|\alpha_{k}\right|\left|\sigma\left(w\left(t-t_{k}\right)\right)\right|
\end{aligned}
$$


for every $t \in I$. Furthermore,

$$
\begin{aligned}
\left|E_{N}^{\sigma}(t)\right| \leq & \frac{(b-a) M_{1}}{N M_{2}}\left(e^{M_{2}(t-a)}-1\right) \\
& +\left|\alpha_{0}\right| \int_{a}^{t}\left|\sigma\left(w\left(s-t_{-1}\right)\right)-1\right| d s \\
& +\sum_{k=1}^{N}\left|\alpha_{k}\right| \int_{a}^{t}\left|\sigma\left(w\left(s-t_{k}\right)\right)\right| d s
\end{aligned}
$$

for every $t \in\left[a, t_{1}\right]$, and

$$
\begin{aligned}
\left|E_{N}^{\sigma}(t)\right| \leq & \frac{(b-a) M_{1}}{N M_{2}}\left(e^{M_{2}(t-a)}-1\right) \\
& +\left|\alpha_{0}\right| \int_{a}^{t}\left|\sigma\left(w\left(s-t_{-1}\right)\right)-1\right| d s \\
& +\sum_{k=1}^{i-1}\left|\alpha_{k}\right|\left[\int_{a}^{t_{k}}\left|\sigma\left(w\left(s-t_{k}\right)\right)\right| d s\right. \\
& \left.+\int_{t_{k}}^{t}\left|\sigma\left(w\left(s-t_{k}\right)\right)-1\right| d s\right] \\
& +\sum_{k=i}^{N}\left|\alpha_{k}\right| \int_{a}^{t}\left|\sigma\left(w\left(s-t_{k}\right)\right)\right| d s .
\end{aligned}
$$

for every $t \in\left[t_{i-1}, t_{i}\right], i=2, \ldots, N$, and $N \in \mathbf{N}^{+}$, where $M_{1}$ and $M_{2}$ are suitable positive constants not depending on $N$.

Proof. We first write $\left(G_{N}^{\sigma} y^{\prime}\right)(t)=:\left(G_{N} y^{\prime}\right)(t)+R_{N}(t)$, where $G_{N} y^{\prime}$ is defined in (4) with the coefficients $\alpha_{k}$ determined in Theorem 3.1, and $R_{N}$ is a suitable function. Let $t \in I:=[a, b]$ be fixed. We have

$$
\begin{aligned}
\left|R_{N}(t)\right|=\left|\left(G_{N}^{\sigma} y^{\prime}\right)(t)-\left(G_{N} y^{\prime}\right)(t)\right| \\
=\mid \sum_{k=1}^{N} \alpha_{k}\left[\sigma\left(w\left(t-t_{k}\right)\right)-H\left(t-t_{k}\right)\right] \\
\quad+\alpha_{0}\left[\sigma\left(w\left(t-t_{-1}\right)\right)-1\right] \mid
\end{aligned}
$$


where $w>0$ is a suitable parameter depending on $\sigma$ and $N \in \mathbf{N}^{+}$. We can observe that $\sigma\left(w\left(t-t_{k}\right)\right)-H\left(t-t_{k}\right)=\sigma\left(w\left(t-t_{k}\right)\right)-1$ if $t \geq t_{k}$ and $\sigma\left(w\left(t-t_{k}\right)\right)-H\left(t-t_{k}\right)=\sigma\left(w\left(t-t_{k}\right)\right)$ if $t<t_{k}$, for every $k=1, \ldots, N$. Thus,

$$
\left|R_{N}(t)\right| \leq \sum_{k: t_{k} \leq t}\left|\alpha_{k}\right|\left|\sigma\left(w\left(t-t_{k}\right)\right)-1\right|+\sum_{k: t_{k}>t}\left|\alpha_{k}\right|\left|\sigma\left(w\left(t-t_{k}\right)\right)\right| .
$$

By the inequality above and Theorem 4.1, we can write

$$
\begin{aligned}
\left|e_{N}^{\sigma}(t)\right|= & \left|y^{\prime}(t)-\left(G_{N}^{\sigma} y^{\prime} t\right)\right| \leq\left|y^{\prime}(t)-\left(G_{N} y^{\prime}\right)(t)\right| \\
& +\left|\left(G_{N} y^{\prime}\right)(t)-\left(G_{N}^{\sigma} y^{\prime}\right)(t)\right| \\
= & \left|e_{N}(t)\right|+\left|R_{N}(t)\right| \leq \frac{(b-a) M_{1}}{N} e^{M_{2}(t-a)} \\
& +\sum_{k: t_{k} \leq t}\left|\alpha_{k}\right|\left|\sigma\left(w\left(t-t_{k}\right)\right)-1\right| \\
& +\sum_{k: t_{k}>t}\left|\alpha_{k}\right|\left|\sigma\left(w\left(t-t_{k}\right)\right)\right|,
\end{aligned}
$$

for every $t \in I$. Finally, we can also obtain

$$
\begin{aligned}
\left|E_{N}^{\sigma}(t)\right| \leq & \int_{a}^{t}\left|e_{N}^{\sigma}(s)\right| d s \leq \frac{(b-a) M_{1}}{N} \int_{a}^{t} e^{M_{2}(s-a)} d s \\
& +\left|\alpha_{0}\right| \int_{a}^{t}\left|\sigma\left(w\left(s-t_{-1}\right)\right)-1\right| d s \\
& +\sum_{k=1}^{N}\left|\alpha_{k}\right| \int_{a}^{t}\left|\sigma\left(w\left(s-t_{k}\right)\right)\right| d s,
\end{aligned}
$$

for every $t \in\left[a, t_{1}\right]$, i.e.,

$$
\begin{aligned}
\left|E_{N}^{\sigma}(t)\right| \leq & \frac{(b-a) M_{1}}{N M_{2}}\left(e^{M_{2}(t-a)}-1\right) \\
& +\left|\alpha_{0}\right| \int_{a}^{t}\left|\sigma\left(w\left(s-t_{-1}\right)\right)-1\right| d s \\
& +\sum_{k=1}^{N}\left|\alpha_{k}\right| \int_{a}^{t}\left|\sigma\left(w\left(s-t_{k}\right)\right)\right| d s
\end{aligned}
$$


for every $t \in\left[a, t_{1}\right]$, where the positive numbers $M_{1}$ and $M_{2}$ are those determined in the proof of Theorem 4.1. Moreover, we have, for $t \in\left(t_{i-1}, t_{i}\right], i=2, \ldots, N$,

$$
\begin{aligned}
\left|E_{N}^{\sigma}(t)\right| \leq & \frac{(b-a) M_{1}}{N} \int_{a}^{t} e^{M_{2}(s-a)} d s \\
& +\left|\alpha_{0}\right| \int_{a}^{t}\left|\sigma\left(w\left(s-t_{-1}\right)\right)-1\right| d s \\
& +\sum_{k=1}^{i-1}\left|\alpha_{k}\right|\left[\int_{a}^{t_{k}}\left|\sigma\left(w\left(s-t_{k}\right)\right)\right| d s\right. \\
& \left.+\int_{t_{k}}^{t}\left|\sigma\left(w\left(s-t_{k}\right)\right)-1\right| d s\right] \\
& +\sum_{k=i}^{N}\left|\alpha_{k}\right| \int_{a}^{t}\left|\sigma\left(w\left(s-t_{k}\right)\right)\right| d s,
\end{aligned}
$$

or, equivalently,

$$
\begin{aligned}
\left|E_{N}^{\sigma}(t)\right| \leq & \frac{(b-a) M_{1}}{N M_{2}}\left(e^{M_{2}(t-a)}-1\right) \\
& +\left|\alpha_{0}\right| \int_{a}^{t}\left|\sigma\left(w\left(s-t_{-1}\right)\right)-1\right| d s \\
& +\sum_{k=1}^{i-1}\left|\alpha_{k}\right|\left[\int_{a}^{t_{k}}\left|\sigma\left(w\left(s-t_{k}\right)\right)\right| d s\right. \\
& \left.+\int_{t_{k}}^{t}\left|\sigma\left(w\left(s-t_{k}\right)\right)-1\right| d s\right] \\
& +\sum_{k=i}^{N}\left|\alpha_{k}\right| \int_{a}^{t}\left|\sigma\left(w\left(s-t_{k}\right)\right)\right| d s .
\end{aligned}
$$

Note that, from the definition itself of sigmoidal functions, the terms $\left|\sigma\left(w\left(t-t_{k}\right)\right)-1\right|$ and $\left|\sigma\left(w\left(t-t_{k}\right)\right)\right|$ in the estimates of Theorem 4.8 are small, when the positive parameter $w$ is sufficiently large.

Now, we apply Theorem 4.8 to the special case of logistic sigmoidal functions. This yields the following 
Corollary 4.9. If $\sigma_{\ell}(t)=\left(1+e^{-t}\right)^{-1}, t \in \mathbf{R}$, and (3) is a given VIDE of the neutral type, having a classical solution, $y \in C^{1}(I)$, we have, under the assumptions (i)-(iv) of Theorem 4.1, for every $w>(N /(b-a)) \ln (N-1)$,

$$
\begin{aligned}
\left|e_{N}^{\sigma}(t)\right| \leq & \frac{(b-a) M_{1}}{N} e^{M_{2}(t-a)} \\
& +\sum_{k: t_{k} \leq t}\left|\alpha_{k}\right| \frac{e^{-w\left(t-t_{k}\right)}}{1+e^{-w\left(t-t_{k}\right)}} \\
& +\sum_{k: t_{k}>t}\left|\alpha_{k}\right|\left(1+e^{-w\left(t-t_{k}\right)}\right)^{-1}
\end{aligned}
$$

for every $t \in I$. Furthermore,

$$
\begin{aligned}
\left|E_{N}^{\sigma}(t)\right| \leq & \frac{(b-a) M_{1}}{N M_{2}}\left(e^{M_{2}(t-a)}-1\right) \\
& +\left\{\left|\alpha_{0}\right| \ln \left(\frac{1+e^{-w(b-a) / N}}{1+e^{-w\left(t-t_{-1}\right)}}\right)\right. \\
& \left.+\sum_{k=1}^{N}\left|\alpha_{k}\right| \ln \left(1+\frac{e^{w(t-a)}}{1+e^{w\left(t_{k}-a\right)}}\right)\right\} \frac{b-a}{N \ln (N-1)},
\end{aligned}
$$

for every $t \in\left[a, t_{1}\right]$, and

$$
\begin{aligned}
\left|E_{N}^{\sigma}(t)\right| \leq & \frac{(b-a) M_{1}}{N M_{2}}\left(e^{M_{2}(t-a)}-1\right)+\left\{\left|\alpha_{0}\right| \ln \left(\frac{1+e^{-w(b-a) / N}}{\left.1+e^{-w(t-t-1}\right)}\right)\right. \\
& +2 \ln 2\left[\sum_{k=1}^{i-1}\left|\alpha_{k}\right|\right] \\
& \left.+\sum_{k=i}^{N}\left|\alpha_{k}\right| \ln \left(1+\frac{e^{w(t-a)}}{1+e^{w\left(t_{k}-a\right)}}\right)\right\} \frac{b-a}{N \ln (N-1)}
\end{aligned}
$$

for every $t \in\left(t_{i-1}, t_{i}\right], i=2, \ldots, N$, and $N \in \mathbf{N}^{+}, N>2$, where $M_{1}$ and $M_{2}$ are suitable positive constants not dependent on $N$. 
Proof. The proof is a direct consequence of Theorem 4.8 and Corollary 2.3 , just observing that

$$
\begin{aligned}
& \int_{a}^{t_{k}}\left|\sigma_{\ell}\left(w\left(s-t_{k}\right)\right)\right| d s+\int_{t_{k}}^{t}\left|\sigma_{\ell}\left(w\left(s-t_{k}\right)\right)-1\right| d s \\
& \leq 2 \ln 2 w^{-1} \leq \frac{2 \ln 2(b-a)}{N \ln (N-1)}
\end{aligned}
$$

for $k=1, \ldots, i-1$, where $i=2, \ldots, N$, is such that $t \in\left(t_{i-1}, t_{i}\right]$, for $w>N /(b-a) \ln (N-1)$, and $N \in \mathbf{N}^{+}, N>2$.

Clearly, the analogue of Corollary 4.9 can be established for the case of the Gompertz sigmoidal functions.

Remark 4.10. Theorem 4.8 and Corollary 4.9 can also be extended to the case of VIDEs like (14) and (15), thus obtaining a posteriori estimates for the corresponding errors, $e_{N}^{\sigma}$ and $E_{N}^{\sigma}$. Further estimates can be obtained replacing the assumptions of Theorem 4.1 with those of Theorem 4.6, in Theorem 4.8 (and in Corollary 4.9).

5. Numerical examples. In this section, we apply the collocation method developed earlier in this paper to numerically solve some VIDEs of the form (3), (14) and (15).

Example 5.1. Consider the nonlinear VIDE, of the form (3),

$$
\begin{aligned}
y^{\prime}(t)= & 2 e^{-t}-e^{-y(t)} \\
& +2 \int_{0}^{t} e^{s-t-y(s)}\left[2-y^{\prime}(s)-\ln \left(e(1+s) y^{\prime}(s)\right)\right] d s,
\end{aligned}
$$

with initial condition $y(0)=0$, for $t \in[0,1]$. Its solution is $y(t)=$ $\ln (1+t)$.

In Table 1, the corresponding numerical errors, obtained by our collocation method with unit step functions, are shown. In the same table, we also show the numerical errors made when logistic functions, $\sigma_{\ell}$, and Gompertz functions, $\sigma_{\alpha \beta}$ with $\alpha=0.85$ and $\beta=0.1$, are used. 
TABLE 1. Numerical results for Example 5.1. $E_{N}, E_{N}^{\sigma_{\ell}}$ and $E_{N}^{\sigma \alpha \beta}$ are the errors on $y$, see Section 4 . The scaling parameters, $w$ of the collocation solutions $S_{N}^{\sigma_{\ell}} y$ and $S_{N}^{\sigma_{\alpha \beta}} y$ are $w=N^{2}$ and $w=N^{2} /(\alpha \beta)$, respectively, see Sections 2 and 3 .

\begin{tabular}{|ccccc|}
\hline$N$ & $\left\|E_{N}\right\|_{\infty}$ & $\left\|E_{N}^{\sigma_{\ell}}\right\|_{\infty}$ & $\left\|E_{N}^{\sigma_{\alpha \beta}}\right\|_{\infty}$ & $1 /(N \ln N)$ \\
\hline 5 & $2.11 \times 10^{-2}$ & $1.86 \times 10^{-2}$ & $2.6 \times 10^{-2}$ & $1.24 \times 10^{-1}$ \\
10 & $9.9 \times 10^{-3}$ & $9.6 \times 10^{-3}$ & $1.13 \times 10^{-2}$ & $4.34 \times 10^{-2}$ \\
15 & $6.4 \times 10^{-3}$ & $6.4 \times 10^{-3}$ & $7.1 \times 10^{-3}$ & $2.46 \times 10^{-2}$ \\
30 & $3.2 \times 10^{-3}$ & $3.2 \times 10^{-3}$ & $3.3 \times 10^{-3}$ & $9.8 \times 10^{-3}$ \\
40 & $2.4 \times 10^{-3}$ & $2.4 \times 10^{-3}$ & $2.5 \times 10^{-3}$ & $6.8 \times 10^{-3}$ \\
100 & $9.44 \times 10^{-4}$ & $9.44 \times 10^{-4}$ & $9.60 \times 10^{-4}$ & $2.2 \times 10^{-3}$ \\
200 & $4.71 \times 10^{-4}$ & $4.71 \times 10^{-4}$ & $4.75 \times 10^{-4}$ & $9.43 \times 10^{-4}$ \\
\hline
\end{tabular}

TABLE 2. Numerical errors for the piecewise polynomial collocation method for the equation in Example 5.1.

\begin{tabular}{|cccccc|}
\hline$N:$ & 5 & 10 & 15 & 30 & 100 \\
\hline Errors: & $1.5 \times 10^{-3}$ & $1.13 \times 10^{-3}$ & $1.11 \times 10^{-3}$ & $6.47 \times 10^{-4}$ & $2.09 \times 10^{-4}$ \\
\hline
\end{tabular}

From the results of Table 1 it seems that the numerical errors pertaining to Example 5.1 decay to zero roughly as $1 /(N \ln N)$, when $N$ gets large, hence faster than shown by the theoretical results given in Section 4.

In order to assess accuracy and performance of our collocation method, based on sigmoidal functions, we compared it with the classical piecewise polynomial collocation method. In what follows, such a comparison is made considering the collocation solutions in the space of piecewise polynomials whose degree does not exceed $m=2$, with collocation parameters $c_{1}=0$ and $c_{2}=1$, i.e., the well-known Lobatto points, see [9]. Here, $h_{N}:=(b-a) / N$ is the uniform mesh size, and $N$ is the number of subintervals of $[a, b]$ where the collocation is accomplished. In Table 2, the absolute errors for such a piecewise collocation method are given, for several values of $N$, for the VIDE of the neutral type in Example 5.1. 
TABLE 3. Comparison between the CPU time (in seconds) for sigmoidal and piecewise collocation methods for the problem in Example 5.1.

\begin{tabular}{|c|c|c|}
\hline$N$ & sigmoidal functions & piecewise polynomial \\
\hline 5 & 0.012895 & 0.069862 \\
10 & 0.037928 & 0.097894 \\
15 & 0.076565 & 0.149001 \\
30 & 0.292096 & 0.314933 \\
\hline
\end{tabular}

TABLE 4. Numerical results for Example 5.1. $e_{N}, e_{N}^{\sigma_{\ell}}$ and $e_{N}^{\sigma_{\alpha \beta}}$ are the errors made computing $y^{\prime}$, see Section 4. The scaling parameters, $w$, of the $G_{N}^{\sigma_{\ell}} y^{\prime}$ and $G_{N}^{\sigma_{\alpha \beta}} y^{\prime}$ are $w=N^{2}$ and $w=N^{2} /(\alpha \beta)$, respectively.

\begin{tabular}{|cccc|}
\hline$N$ & $\left\|e_{N}\right\|_{\infty}$ & $\left\|e_{N}^{\sigma_{\ell}}\right\|_{\infty}$ & $\left\|e_{N}^{\sigma_{\alpha \beta}}\right\|_{\infty}$ \\
\hline 5 & $1.50 \times 10^{-1}$ & $8.37 \times 10^{-2}$ & $1.25 \times 10^{-1}$ \\
10 & $8.26 \times 10^{-2}$ & $6.17 \times 10^{-2}$ & $7.6 \times 10^{-2}$ \\
15 & $5.66 \times 10^{-2}$ & $4.60 \times 10^{-2}$ & $5.61 \times 10^{-2}$ \\
30 & $2.91 \times 10^{-2}$ & $2.75 \times 10^{-2}$ & $2.91 \times 10^{-2}$ \\
40 & $1.96 \times 10^{-2}$ & $1.96 \times 10^{-2}$ & $1.96 \times 10^{-2}$ \\
100 & $1.9 \times 10^{-3}$ & $4.9 \times 10^{-3}$ & $5.6 \times 10^{-3}$ \\
200 & $9.70 \times 10^{-4}$ & $2.4 \times 10^{-3}$ & $2.8 \times 10^{-3}$ \\
\hline
\end{tabular}

TABLE 5. Numerical results for Example 5.2. $E_{N}, E_{N}^{\sigma_{\ell}}$ and $E_{N}^{\sigma_{\alpha \beta}}$ with $\alpha=0.85$ and $\beta=0.1$ are the errors made evaluating $y$. The scaling parameters, $w$, of $S_{N}^{\sigma_{\ell}} y$ and $S_{N}^{\sigma_{\alpha \beta}} y$ are $w=N^{2}$ and $w=N^{2} /(\alpha \beta)$, respectively.

\begin{tabular}{|ccccc|}
\hline$N$ & $\left\|E_{N}\right\|_{\infty}$ & $\left\|E_{N}^{\sigma_{\ell}}\right\|_{\infty}$ & $\left\|E_{N}^{\sigma_{\alpha \beta}}\right\|_{\infty}$ & $1 /(N \ln N)$ \\
\hline 5 & $4.1 \times 10^{-3}$ & $3.6 \times 10^{-3}$ & $4.6 \times 10^{-3}$ & $1.24 \times 10^{-1}$ \\
10 & $1.8 \times 10^{-3}$ & $1.8 \times 10^{-3}$ & $2 \times 10^{-3}$ & $4.34 \times 10^{-2}$ \\
15 & $1.2 \times 10^{-3}$ & $1.2 \times 10^{-3}$ & $1.3 \times 10^{-3}$ & $2.46 \times 10^{-2}$ \\
30 & $5.73 \times 10^{-4}$ & $5.71 \times 10^{-4}$ & $5.92 \times 10^{-4}$ & $9.8 \times 10^{-3}$ \\
40 & $4.26 \times 10^{-4}$ & $4.25 \times 10^{-4}$ & $4.37 \times 10^{-4}$ & $6.8 \times 10^{-3}$ \\
100 & $1.67 \times 10^{-4}$ & $1.67 \times 10^{-4}$ & $1.69 \times 10^{-4}$ & $2.2 \times 10^{-3}$ \\
\hline
\end{tabular}

TABLE 6. Numerical errors for the piecewise polynomial collocation method for the equation in Example 5.2.

\begin{tabular}{|cccccc|}
\hline$N:$ & 5 & 10 & 15 & 30 & 100 \\
\hline Errors: & $5.44 \times 10^{-2}$ & $1.7 \times 10^{-2}$ & $9 \times 10^{-3}$ & $3.1 \times 10^{-3}$ & $5.03 \times 10^{-4}$ \\
\hline
\end{tabular}


Comparing the numerical errors in Table 2 with those in Table 1, we see, in general, the piecewise polynomial collocation methods, which are local in nature, are more accurate than the method based on sigmoidal functions. Moreover, for the piecewise polynomial collocation method, $m N=2 N$ collocation points are needed, where $N$ is the number of subintervals of $[a, b]$. We should then compare the numerical results in Table 2 with those of Table 1, when the same number of collocation points is used.

However, our method is simpler and offers some computational advantages. Indeed, to solve nonlinear equations, we do not need to compute the solutions of a sequence of nonlinear algebraic systems, as it happens using the piecewise polynomial collocation method. To determine the coefficients of our collocation solutions, we can merely apply the explicit linear recursive formulae given in Theorem 3.1. All integrals in such formulae can be computed by numerical quadrature. In Table 3, we show the CPU times needed to compute the solutions with the sigmoidal and piecewise polynomial collocation methods for Example 5.1, for various $N$.

Comparing the CPU times given in Table 3 we can observe that the numerical solutions by the collocation method with sigmoidal functions are computed in a shorter time than the piecewise polynomial collocation method.

In addition, our method provides a simultaneous approximation of the first derivative of the solution by a superposition of sigmoidal functions. In Example 5.1, $y^{\prime}(t)=(1+t)^{-1}, t \in[0,1]$. In Table 4, the numerical errors made in the approximation of $y^{\prime}$ with $G_{N} y^{\prime}, G_{N}^{\sigma_{\ell}} y^{\prime}$ and $G_{N}^{\sigma_{\alpha \beta}} y^{\prime}$, $\alpha=0.85$ and $\beta=0.1$ are shown.

Here the numerical errors in the approximation of $y^{\prime}$ are larger than those made approximating $y$.

Example 5.2. Consider the following classical nonlinear VIDE like (14), with a weakly singular kernel, $K$, i.e.,

$$
y^{\prime}(t)=\frac{1}{8 y(t)}+\sqrt{t}\left(\frac{t}{3}+\frac{1}{2}\right)-\int_{0}^{t} \frac{y^{2}(s)}{\sqrt{t-s}} d s,
$$

with initial condition $y(0)=1 / 2$, for $t \in[0,1]$. Its solution is $y(t)=(\sqrt{t+1}) / 2$. 
Weakly singular kernels are not Lipschitz continuous near the rightpoints of the interval $[0, t]$, with $t \in[0,1]$. This fact represents a problem for the convergence of the numerical method. The numerical errors for such an example are shown in Table 5.

In Example 5.2, the numerical errors seem to decay roughly as $1 /(N \ln N)$, as well as in Example 5.1.

In Example 5.2, again, it is more interesting to compare the numerical errors of Table 5 with those obtained by piecewise collocation. In Table 6, we show the absolute errors of the piecewise polynomial method applied to the equation in Example 5.2 on $[0,1]$.

Comparing the results in Tables 5 and 6 , we can observe that the collocation method with sigmoidal functions is more accurate than the piecewise polynomial collocation method. Therefore, our numerical method seems to be competitive in the case of equations with weakly singular kernels.

Example 5.3. We consider the non-standard VIDE of the form (15),

$$
y^{\prime}(t)=y(t)\left(\widetilde{f}(t)+\int_{0}^{t} e^{-(t-s)} y(s) d s\right),
$$

where

$$
\widetilde{f}(t):=e^{-2 t}-e^{-t}-2,
$$

and initial condition $y(0)=1, t \in[0,1]$, whose solution is $y(t)=e^{-2 t}$, see $[\mathbf{1 1}]$.

The corresponding numerical errors are given in Table 7 .

Again, in Example 5.3, the same observation made for Examples 5.1 and 5.2 applies, concerning the decay rate of the numerical errors. Here, $\left\|E_{N}\right\|_{\infty},\left\|E_{N}^{\sigma_{\ell}}\right\|_{\infty}$ and $\left\|E_{N}^{\sigma_{\alpha \beta}}\right\|_{\infty}$ seem to decrease as $C /(N \ln N)$, since $C>1$ a suitable constant.

Similar considerations can be made for Example 5.3 as for Example 5.1. In Table 8, the numerical errors obtained using piecewise polynomial collocation on $[0,1]$ are given. Also in this case, the errors of Table 8 turn out to be smaller then those in Table 7 . 
TABLE 7. Numerical results for Example 5.3. $E_{N}, E_{N}^{\sigma_{\ell}}$ and $E_{N}^{\sigma_{\alpha \beta}}$ with $\alpha=0.85$ and $\beta=0.1$ are the errors made evaluating $y$. The scaling parameters, $w$, of $S_{N}^{\sigma_{\ell}} y$ and $S_{N}^{\sigma_{\alpha \beta}} y$ are $w=N^{2}$ and $w=N^{2} /(\alpha \beta)$, respectively.

\begin{tabular}{|cccc|}
\hline$N$ & $\left\|E_{N}\right\|_{\infty}$ & $\left\|E_{N}^{\sigma_{\ell}}\right\|_{\infty}$ & $\left\|E_{N}^{\sigma_{\alpha \beta}}\right\|_{\infty}$ \\
\hline 5 & $8.99 \times 10^{-2}$ & $7.85 \times 10^{-2}$ & $1.01 \times 10^{-1}$ \\
10 & $4.09 \times 10^{-2}$ & $3.98 \times 10^{-2}$ & $4.47 \times 10^{-2}$ \\
15 & $2.63 \times 10^{-2}$ & $2.61 \times 10^{-2}$ & $2.82 \times 10^{-2}$ \\
30 & $1.29 \times 10^{-2}$ & $1.28 \times 10^{-2}$ & $1.34 \times 10^{-2}$ \\
40 & $9.6 \times 10^{-3}$ & $9.6 \times 10^{-3}$ & $9.9 \times 10^{-3}$ \\
100 & $3.8 \times 10^{-3}$ & $3.8 \times 10^{-3}$ & $3.8 \times 10^{-3}$ \\
200 & $1.9 \times 10^{-3}$ & $1.9 \times 10^{-3}$ & $1.9 \times 10^{-3}$ \\
\hline
\end{tabular}

TABLE 8. Numerical errors for the piecewise polynomial collocation method for the equation in Example 5.3.

\begin{tabular}{|cccccc|}
\hline$N:$ & 5 & 10 & 15 & 30 & 100 \\
\hline Errors: & $1.33 \times 10^{-2}$ & $6.40 \times 10^{-3}$ & $4.2 \times 10^{-3}$ & $2.1 \times 10^{-3}$ & $6.34 \times 10^{-4}$ \\
\hline
\end{tabular}

Example 5.4. Consider the nonlinear VIDE of the neutral type:

$$
\begin{aligned}
y^{\prime}(t)= & 2 t^{3}-2 y^{2}(t)-6 t^{2}+13 t+12 e^{-t}-12 \\
& +\int_{0}^{t} e^{t-s} y(s)\left(y^{\prime}(s)\right)^{2} d s
\end{aligned}
$$

for $t \in[0,1]$, subject to the initial condition $y(0)=0$. Its solution is $y(t)=(1 / 2) t^{2}$.

The numerical errors for such an example are shown in Table 9.

In Example 5.4, we can determine all parameters required to obtain the a posteriori error estimates of subsection 4.2. The constants $M_{1}$ and $M_{2}$ in Theorems 4.1 and 4.6 (which depend neither on the number $N$ of sigmoidal functions nor on the mesh size, $h$ ) are

$$
M_{1}=M+C+L_{1}, \quad M_{2}=2 L_{2}+L_{f},
$$


TABLE 9. Numerical results for Example 5.4. $E_{N}, E_{N}^{\sigma_{\ell}}$ and $E_{N}^{\sigma_{\alpha \beta}}$ with $\alpha=0.85$ and $\beta=0.1$ are the errors made evaluating $y$. The scaling parameters, $w$, of $S_{N}^{\sigma_{\ell}} y$ and $S_{N}^{\sigma_{\alpha \beta}} y$ are $w=N^{2}$ and $w=N^{2} /(\alpha \beta)$, respectively.

\begin{tabular}{|cccc|}
\hline$N$ & $\left\|E_{N}\right\|_{\infty}$ & $\left\|E_{N}^{\sigma_{\ell}}\right\|_{\infty}$ & $\left\|E_{N}^{\sigma_{\alpha \beta}}\right\|_{\infty}$ \\
\hline 5 & $6.28 \times 10^{-2}$ & $2.59 \times 10^{-2}$ & $5.57 \times 10^{-2}$ \\
10 & $5 \times 10^{-3}$ & $3.9 \times 10^{-3}$ & $6.8 \times 10^{-3}$ \\
15 & $2.8 \times 10^{-3}$ & $2.5 \times 10^{-3}$ & $3.9 \times 10^{-3}$ \\
30 & $1.4 \times 10^{-3}$ & $1.3 \times 10^{-3}$ & $1.6 \times 10^{-3}$ \\
40 & $1 \times 10^{-3}$ & $1 \times 10^{-3}$ & $1.1 \times 10^{-3}$ \\
100 & $4.07 \times 10^{-4}$ & $4.06 \times 10^{-4}$ & $4.21 \times 10^{-4}$ \\
200 & $2.03 \times 10^{-4}$ & $2.03 \times 10^{-4}$ & $2.07 \times 10^{-4}$ \\
\hline
\end{tabular}

TABLE 10. Numerical errors for the piecewise polynomial collocation method for the equation in Example 5.4.

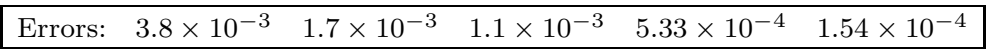

TABLE 11. Comparison between the numerical errors $\left|E_{N}(t)\right|$ and the a posteriori error estimates $R_{N}(t)$ of Theorem 4.6, for Example 5.4.

\begin{tabular}{|c|c|c|c|}
\hline$N$ & $t$ & $\left|E_{N}(t)\right|$ & $R_{N}(t)$ \\
\hline 5 & 0.05 & 0.0013 & 0.2106 \\
5 & 0.07 & 0.0025 & 0.6466 \\
\hline 30 & 0.05 & $5.8332 \times 10^{-4}$ & 0.0351 \\
30 & 0.07 & $8.5885 \times 10^{-4}$ & 0.1078 \\
30 & 0.1 & 0.0011 & 0.5507 \\
\hline 40 & 0.05 & $5.3125 \times 10^{-4}$ & 0.0263 \\
40 & 0.1 & $7.9136 \times 10^{-4}$ & 0.4130 \\
\hline 100 & 0.05 & $1.9677 \times 10^{-4}$ & 0.0105 \\
100 & 0.1 & $3.0052 \times 10^{-4}$ & 0.1652 \\
100 & 0.13 & $3.3732 \times 10^{-4}$ & 0.8315 \\
\hline
\end{tabular}

where $M=\sqrt{L_{y}^{2}+1}=\sqrt{2}, C=L_{1}=e / 2, L_{2}=2 e$, and $L_{f}=16$, hence,

$$
M_{1}=\sqrt{2}+e, \quad M_{2}=4 e+16 .
$$


Moreover, the functions introduced in conditions (a) and (b) of Theorem 4.6 are $\mathcal{L}_{f}(\gamma)=\gamma^{2}$ and $\mathcal{L}_{K}(\gamma)=\gamma^{3}, \gamma \geq 0$, and finally

$$
\gamma_{N}:=\max \left\{1,\left\|S_{N} y\right\|_{\infty},\left\|G_{N} y^{\prime}\right\|_{\infty}\right\}
$$

and,

$$
\mathcal{M}_{N}=\frac{(4 e+16)}{N}\left(\gamma_{N}+\gamma_{N}^{2}\right)
$$

Therefore, the a posteriori estimate provided by Theorem 4.6 for the collocation solutions of such neutral equations is given by

$$
\left|E_{N}(t)\right| \leq \frac{(\sqrt{2}+e)}{N \mathcal{M}_{N}}\left(e^{\mathcal{M}_{N} t}-1\right)=: R_{N}(t),
$$

for every $t \in[0,1]$. This estimate is very sharp, especially when $t$ is near to zero. In Table 11, a comparison between the numerical errors $\left|E_{N}(t)\right|$ and the a posteriori error estimates $R_{N}(t)$ of Theorem 4.6, for Example 5.4, is given. As a rule, a priori estimates provide overestimates for the numerical errors. Clearly, since in the error inequality an exponential function with exponent $\mathcal{M}_{N} t$ appears depending on the (large) constant $\mathcal{M}_{N}$, for larger values of $t$ the previous estimate cannot be very sharp. Similar considerations can be made in the case of an a posteriori estimate obtained approximating solutions to neutral integro-differential equations by logistic or Gompertz functions.

\section{REFERENCES}

1. A.V. Adrianov, An analog of the Jackson-Nikol'skij theorem on the approximation by superposition of sigmoidal functions, Math. Notes 59 (1996), 661-664.

2. M. Aguilar and H. Brunner, Collocation method for second-order Volterra integro-differential equations, Appl. Numer. Math. 4 (1988), 455-470.

3. G. Albano and V. Giorno, On the first exit time problem for a Gompertz-type tumor growth, Lect. Notes Comp. Sci. 5717/2009 (2009), 113-120.

4. K.E. Atkinson, The numerical solution of integral equations of the second kind, Cambridge Mono. Appl. Comp. Math. 4, Cambridge University Press, Cambridge, 1997.

5. M.A. Aves, P.J. Davies and D.J. Higham, Fixed points and spurious modes of a nonlinear infinite-step map, in Numerical analysis: A.R. Mitchel 75th birthday volume, D.F. Griffiths and G.A. Watson, eds., Word Scientific Publishers, Singapore, 1996.

6. - The effect of quadrature on the dynamics of a discretized nonlinear integro-differential equation, Appl. Numer. Math. 32 (2000), 1-20. 
7. A.R. Barron, Universal approximation bounds for superpositions of a sigmoidal function, IEEE Trans. Inform. Theory 39 (1993), 930-945.

8. F. Brauer and C. Castillo Chavez, Mathematical models in population biology and epidemiology, Springer, New York, 2001.

9. H. Brunner, Collocation methods for Volterra integral and related functional equations, Cambridge Mono. Appl. Comp. Math. 15, Cambridge University Press, Cambridge, 2004.

10. - High-order collocation methods for singular Volterra functional equations of neutral type, Appl. Numer. Math. 57 (2007), 533-548.

11. H. Brunner and J. Ma, A posteriori error estimates of discontinuous Galerkin methods for non-standerd Volterra integro-differential equations, IMA J. Numer. Anal. 26 (2006), 78-95.

12. H. Brunner, A. Pedas and G. Vainikko, A spline collocation method for linear Volterra integro-differential equations with weakly singular kernels, BIT 41 (2001), 891-900.

13. - Piecewise polynomial collocation method for linear Volterra integrodifferential equations with weakly singular kernels, SIAM J. Numer. Anal. 39 (2001), 957-982.

14. M. Buhmann and A. Iserles, On the dynamics of a discretized neutral equation, IAM J. Numer. Anal. 12 (1992), 339-363.

15. Debao Chen, Degree of approximation by superpositions of a sigmoidal function, Approx. Theory Appl. 9 (1993), 17-28.

16. H. Chen, T. Chen and R. Liu, A constructive proof and an extension of Cybenko's approximation theorem, Comp. Sci. Stat., Springer-Verlag, New York (1992), 163-168.

17. E.S. Chumerina, Choice of optimal strategy of tumor chemotherapy in Gompertz model, J. Comp. Syst. Sci. Inter. 48 (2009), 325-331.

18. D. Costarelli and R. Spigler, Constructive approximation by superposition of sigmoidal functions, Anal. Theor. Appl. 29 (2013), 169-196.

19. - Solving Volterra integral equations of the second kind by sigmoidal functions approximation, J. Integral Equat. Appl. 25 (2013), 193-222.

20. - Approximation by series of sigmoidal functions with applications to neural networks, to appear in Annal. Mat. Pura Appl. 2013, doi:10.1007/s10231013-0378-y.

21. - Solving numerically nonlinear systems of balance laws by multivariate sigmoidal functions approximation, submitted.

22. J.M. Cushing, Integrodifferential equations and delay models in population dynamics, Lect. Notes Biomath. 20 (1977), Springer-Verlag, Berlin.

23. G. Cybenko, Approximation by superpositions of a sigmoidal function, Math. Contr. Sig. Syst. 2 (1989), 303-314.

24. B. Gao and $\mathrm{Y} . \mathrm{Xu}$, Univariant approximation by superpositions of a sigmoidal function, J. Math. Anal. Appl. 178 (1993), 221-226.

25. N. Hahm and B. Hong, Approximation order to a function in $\bar{C}(\mathbf{R})$ by superposition of a sigmoidal function, Appl. Math. Lett. 15 (2002), 591-597. 
26. N. Hritonenko and Y. Yatsenko, Mathematical modelling in economics, ecology and the environment, Science Press, Beijing, 2006.

27. L.K. Jones, Constructive approximations for neural networks by sigmoidal functions, Proc. IEEE 78 (1990), 1586-1589.

28. G. Lewicki and G. Marino, Approximation by superpositions of a sigmoidal function, Z. Anal. Anwend. J. Anal. Appl. 22 (2003), 463-470.

29. - Approximation of functions of finite variation by superpositions of a sigmoidal function, Appl. Math. Lett. 17 (2004), 1147-1152.

30. W. Light, Ridge functions, sigmoidal functions and neural networks, Academic Press, Boston, MA, 1993.

31. E.A. Mantysaari, M.L. Sevon Aimonen, I. Stranden and K. Vuori, Estimation of non-linear growth models by linearization: A simulation study using a Gompertz function, Genet. Sel. Evol. 38 (2006), 343-358.

32. H.N. Mhaskar and C.A. Micchelli, Approximation by superposition of sigmoidal and radial basis functions, Adv. Appl. Math. 13 (1992), 350-373.

33. R.K. Miller, Nonlinear Volterra integral equations, W.A. Benjamin, Menlo Park, CA, 1971.

34. B.G. Pachpatte, Implicit type Volterra integrodifferential equation, Tamkang J. Math. 41 (2010), 97-107.

35. - Approximate solutions for integrodifferential equations of the neutral type, Comment. Math. Univ. Carolin. 51 (2010), 489-501.

36. A. Pinkus, Approximation theory of the MLP model in neural networks, Acta Numer. 8 (1999), 143-195.

37. A.A. Sas, H. Snieder and J. Korf, Gompertz' survivorship law as an intrinsic principle of aging, Medical Hypotheses (2012), doi:10.1016/j.mehy.2012.02.004.

Department of Mathematics and Physics, Section of Mathematics, University "Roma Tre" 1, Largo S. Leonardo Murialdo, 00146 Rome, Italy Email address: danilo.costarelli@libero.it, costarel@mat.uniroma3.it

Department of Mathematics and Physics, Section of Mathematics, University "Roma Tre" 1, Largo S. Leonardo Murialdo, 00146 Rome, Italy Email address: respigler@gmail.com, spigler@mat.uniroma3.it 